\title{
A Review of Portable High-Performance Liquid Chromatography: the Future of the Field?
}

\author{
Faraz Rahimi $^{1,2} \cdot$ Stelios Chatzimichail $^{1} \cdot$ Aliyah Saifuddin $^{1,2} \cdot$ Andrew J. Surman $^{2} \cdot$ Simon D. Taylor-Robinson ${ }^{1}$. \\ Ali Salehi-Reyhani ${ }^{1}[$
}

Received: 7 May 2020 / Revised: 22 July 2020 / Accepted: 5 August 2020 / Published online: 15 August 2020

(c) The Author(s) 2020

\begin{abstract}
There is a growing need for chemical analyses to be performed in the field, at the point of need. Tools and techniques often found in analytical chemistry laboratories are necessary in performing these analyses, yet have, historically, been unable to do so owing to their size, cost and complexity. Technical advances in miniaturisation and liquid chromatography are enabling the translation of these techniques out of the laboratory, and into the field. Here we examine the advances that are enabling portable liquid chromatography (LC). We explore the evolution of portable instrumentation from its inception to the most recent advances, highlighting the trends in the field and discussing the necessary criteria for developing in-field solutions. While instrumentation is becoming more capable it has yet to find adoption outside of research.
\end{abstract}

Keywords Portable HPLC $\cdot$ Miniaturised chromatography $\cdot$ Field chemistry

\section{Introduction}

\section{Field-Based Chemistry}

The miniaturisation and translation of laboratory-based analytical methods to the field is a major endeavour in instrumentation development. Researchers from various fields have made significant progress towards this goal with a variety of technologies. The aim of this review is to bring attention to the advances in miniaturised high-performance liquid chromatography (HPLC) systems and the endeavours to bring the laboratory to the sample.

The realisation of other field-based chemistry has made a major impact on a variety of societal challenges. For instance, outbreaks of foot-and-mouth disease virus

Electronic supplementary material The online version of this article (https://doi.org/10.1007/s10337-020-03944-6) contains supplementary material, which is available to authorized users.

Ali Salehi-Reyhani

ali.salehi-reyhani@imperial.ac.uk

1 Department of Surgery and Cancer, Imperial College London, London, UK

2 Department of Chemistry, King's College London, London, UK
(FMDV) infect livestock globally resulting in extreme economic loss [1]. Wildlife disease surveillance focusses on early detection, vital to minimise the damage caused by FMDV outbreaks. The evolution of portable platforms capable of real-time reverse transcription polymerase chain reaction (RRT-PCR) and amplification assays for FMDV eliminate the delays associated with transportation of samples to the laboratory [2]. The Biomeme range of instruments (Biomeme Inc., Philadelphia, PA, USA), and similar, have revolutionised surveillance in sectors ranging from aquatic ecology to industrial quality control, allowing informed decision-making based on developments in real-time. Franklin ${ }^{\mathrm{TM}}$ by Biomeme (Fig. 1) is a handheld quantitative PCR thermocycler able to deliver results from multiple samples in less than an hour and display these on a mobile-based app for rapid identification. This instrument, weighing around only $1 \mathrm{~kg}$, is a striking example of modern analytical devices with designed for field applications.

Groundwater extracted from tube wells in Bangladesh acts as the main source of drinking and irrigation water for millions of residents. This has mainly been due to the severe issues with infectious diseases arising from contaminants in centralised water supplies. While the number of water-borne infections decreased, several forms of cancer were characterised resulting from arsenic contamination of groundwater from the tube wells [4]. Accordingly, accurate 


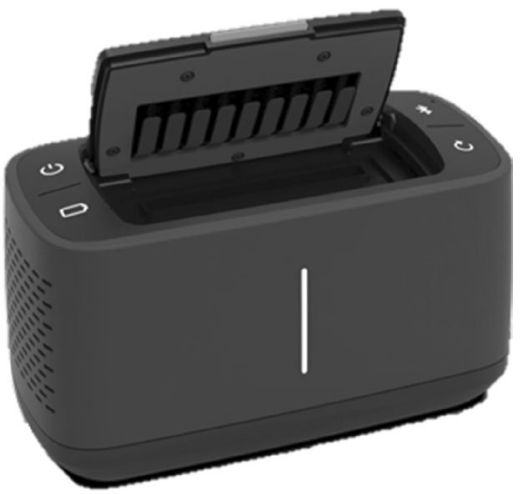

Fig. 1 The Franklin ${ }^{\mathrm{TM}}$ by Biomeme handheld quantitative PCR platform (Biomeme Inc., Philadelphia, PA, USA). Reproduced from Biomeme [3]

and efficient arsenic testing methods that can be carried out by civilians is required. The digital arsenic testing kit from Palintest (Palintest, Gateshead, United Kingdom), known as the Arsenator, enables arsenic detection in liquids with sensitivities in the parts-per-billion (ppb) region. The kit consists of reagents which reduce arsenic to arsine gas transforming the colour of a mercury bromide filter strip on reaction. The Arsenator serves as a digital spectrophotometer to determine the arsenic levels based on the hue of the filter strip. The complete procedure can be performed in $20 \mathrm{~min}$ [5]. The availability of this technology represents a shift in the scientific knowledge required by the end user. By making the chemistry behind arsenic testing more accessible and packaging the solution into easy-to-follow steps, the civilian population can access clean drinking water with total arsenic content within the World Health Organisation guideline concentrations.

Numerous high impact food fraud and contamination cases have occurred worldwide, a selection of which include the horsemeat scandal in 2013 which spread across Europe;
China's melamine-laced milk scandal in 2008; and the contamination of Irish pork products with dioxins in animal feed in 2008 [6]. These represent only a small number of the major incidents within the ongoing crisis around adulterated and contaminated food. Adulterated food products are a global health hazard and are the cause of billions of pounds in lost revenue every year in the UK. Fourier Transform-Infrared Spectroscopy (FTIR) is a standard addition to any research laboratory and has contributed to ongoing food ingredient authentication [7-10]. Handheld Attenuated Total Reflectance (ATR) FTIR spectrometers have steadily been commercialised over the past decade, an example of which is the Agilent 4300 Handheld FTIR (Agilent, Santa Clara, CA, USA). This device has become increasingly popular as an analytical tool due to the light weight $(2 \mathrm{~kg})$ and ergonomic design (Fig. 2) [11].

Similarly, near infrared (NIR) spectrometers have also become commercially available in portable and handheld designs. One such example is the MicroNIR family of spectrometers (VIAVI Solutions Inc, San Jose, CA, USA) with the smallest offering weighing less than $400 \mathrm{~g}$ and a footprint similar to a tennis ball. Two notable application areas are as part of quality control testing; and to identify counterfeit pharmaceuticals. One such example is the ongoing struggle to discriminate between authentic and illegal erectile dysfunction medication at border control. The MicroNIR spectrometer can be used to effectively differentiate between genuine and counterfeit pharmaceuticals (Fig. 3) [12, 13].

Raman spectroscopy has also kept up with the trend towards miniaturisation in the field of analytical chemistry. Several handheld Raman spectrometers have been developed and commercialised in recent years (Fig. 4) [14-17]. Over the past two decades, tragic terrorist attacks have created the need to identify locations where explosives, chemical warfare agents, and precursors to these materials are manufactured and stored. Law enforcement agencies, forensic investigators, and the military require rapid identification
Fig. 2 Agilent 4300 Handheld FTIR spectrometer (Agilent, Santa Clara, CA, USA) a image, b practical use in geological studies. Reproduced from Agilent [11] $\mathbf{a}$

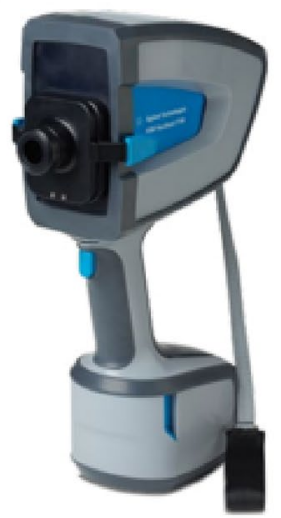

b

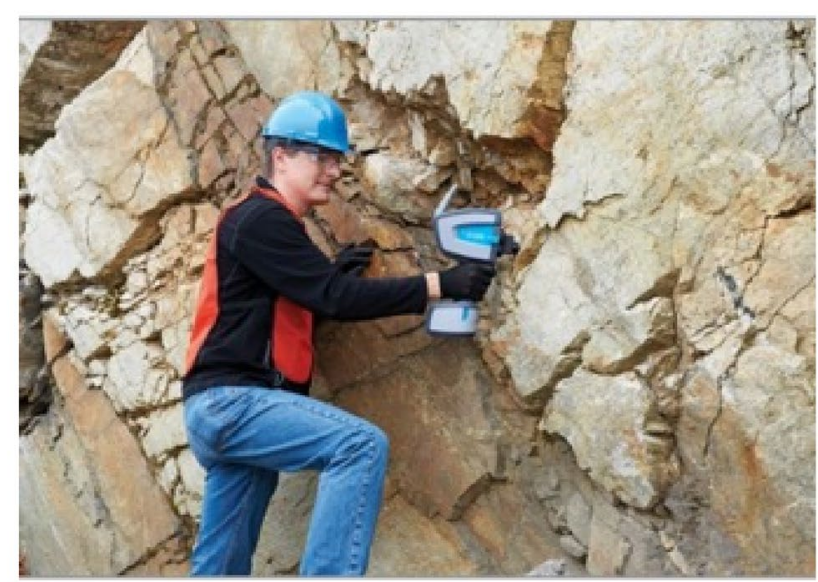


Fig. 3 a The MicroNIR spectrometer used in the study (VIAVI Solutions Inc, San Jose, CA, USA). b The absorbance NIR spectra of the authentic and counterfeit $(\mathrm{CF})$ Alli ${ }^{\circledR}$ drug. Reproduced from Alcalà et al. [13] a

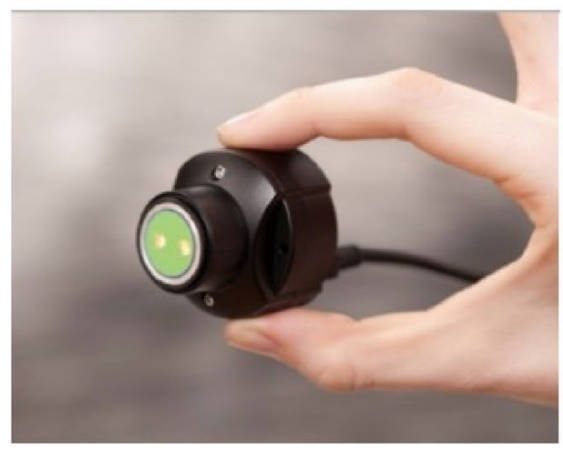

b

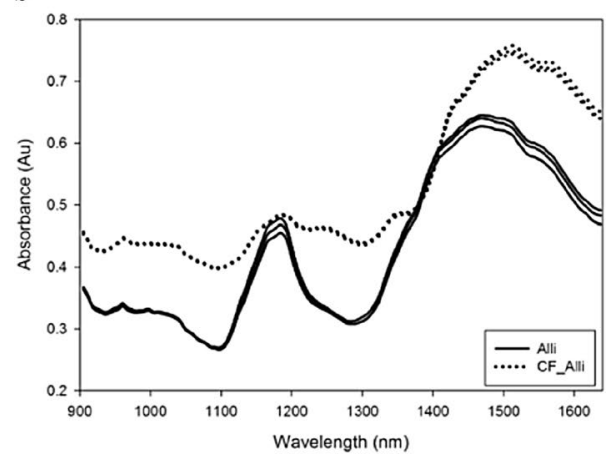

Fig. 4 Images of a Anton Paar handheld Raman spectrometer: Cora 100 (Anton Paar GmbH, Graz, Austria) [15], b Mira DS handheld Raman spectrometer (Metrohm UK Ltd, Runcorn, United Kingdom) [17]
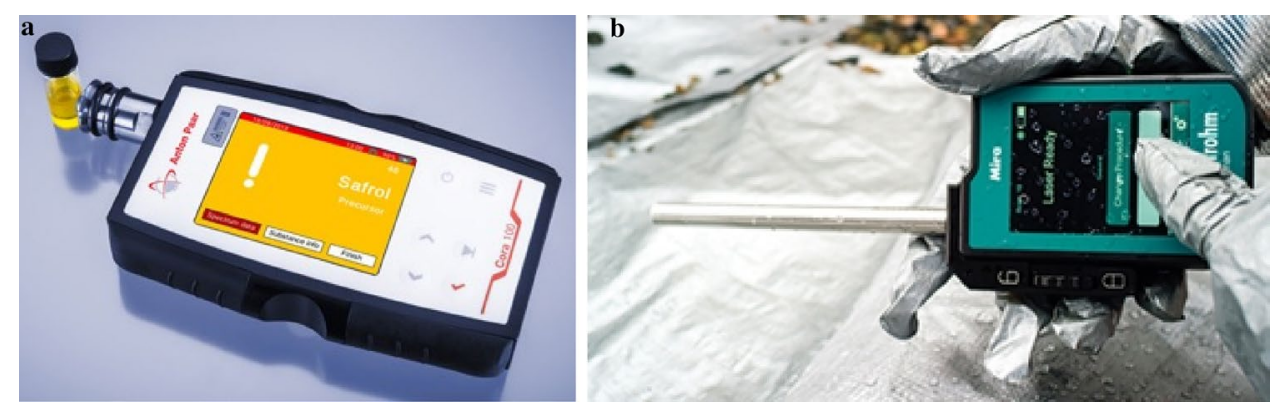

of unidentified materials within clandestine laboratories and military operations as well as crime scenes to determine threats in a timely manner [18]. This class of situations demand fast qualitative results to identify the presence of dangerous materials. Less emphasis is placed on quantitation since the identification of such threats will determine if further sampling is necessary or if the operator should retreat to safety immediately. With the advantage of minimal sample preparation, the commercial availability of handheld Raman spectrometers has transformed the ability of law enforcement to ensure the safety of the public.

Microfluidic devices are another example of the miniaturisation of bench-top methods and instrumentation. By exploring the properties of liquids at the microscale, researchers are able to exploit large scale applications within a portable footprint. Ngamsom et al. [19] developed a microfluidic system for the screening of E. coli $\mathrm{O} 157: \mathrm{H} 7$ in water analysis. E. coli $\mathrm{O} 157: \mathrm{H} 7$ is a foodborne pathogen thriving within cattle and causing widespread disease in humans [20]. Laboratory-based PCR methods are the common route to detection of this bacterial strain; however, high capital costs for instrumentation and qualified personnel impede the application of these methods in resource-poor regions. The portable and inexpensive microfluidic system developed in this research allows semi-skilled personnel to detect the strain within 20 min using a fully integrated system which can be used at the point-of-sampling.

In some cases, demands on field instruments can be extreme. In the coming years, the ExoMars mission, a joint project between the European and Russian space agencies, will be pioneering the investigation into the molecular signs of life on the red planet. The Rosalind Franklin rover (Fig. 5) will house the Mars Organic Molecular Analyzer (MOMA), a combined laser desorption/gas chromatography-mass spectrometry system [21]. The primary goal of the rover's mission will be to uncover the presence of complex organic compounds, molecular signs of past or present life on Mars. Of course, this review will focus on MOMA's terrestrial counterparts; however, the robotic exploration of other world's in our solar system is an incredible example to illustrate what is possible for the portability and miniaturisation of analytical instrumentation. Furthermore, staying on the Red Planet, what is equally, if not more, important is to consider the analytical process in the field as a whole; in the case of MOMA, an important complication is sample preparation. Organic molecules exposed on the surface of the Martian terrain can be gradually destroyed from the thin atmosphere, with no magnetic field to protect them from solar radiation. Therefore, the rover will acquire samples below the surface with a coring drill. Rock fragments and powder are dropped into a crushing station which grinds the sample to a powder with a desired particle size distribution. The dosing mechanism then feeds one of the on-board analysers, including MOMA. In far-flung 'fields' there is no opportunity to repair or replenish either the instruments or reagents: everything, including blank (organically inert) samples, must be accounted for and on board. Robotic explorers are becoming increasingly capable analytical 
Fig. 5 The Rosalind Franklin rover with MOMA instrumentation to be used in the ExoMars 2022 mission. The different parts of MOMA and their contributors (France $(\mathrm{F})$, Germany (D), United States). The LPU that controls the LH is mounted as a slice on the MEB (top right). Sample carousel (SC) and refillable sample container are not part of MOMA. LH, Laser Head; LPU, Laser Pump Unit; MEB, Main Electronic Box; MOMA, Mars Organic Molecule Analyzer. Reproduced from Goesmann et al. [21]

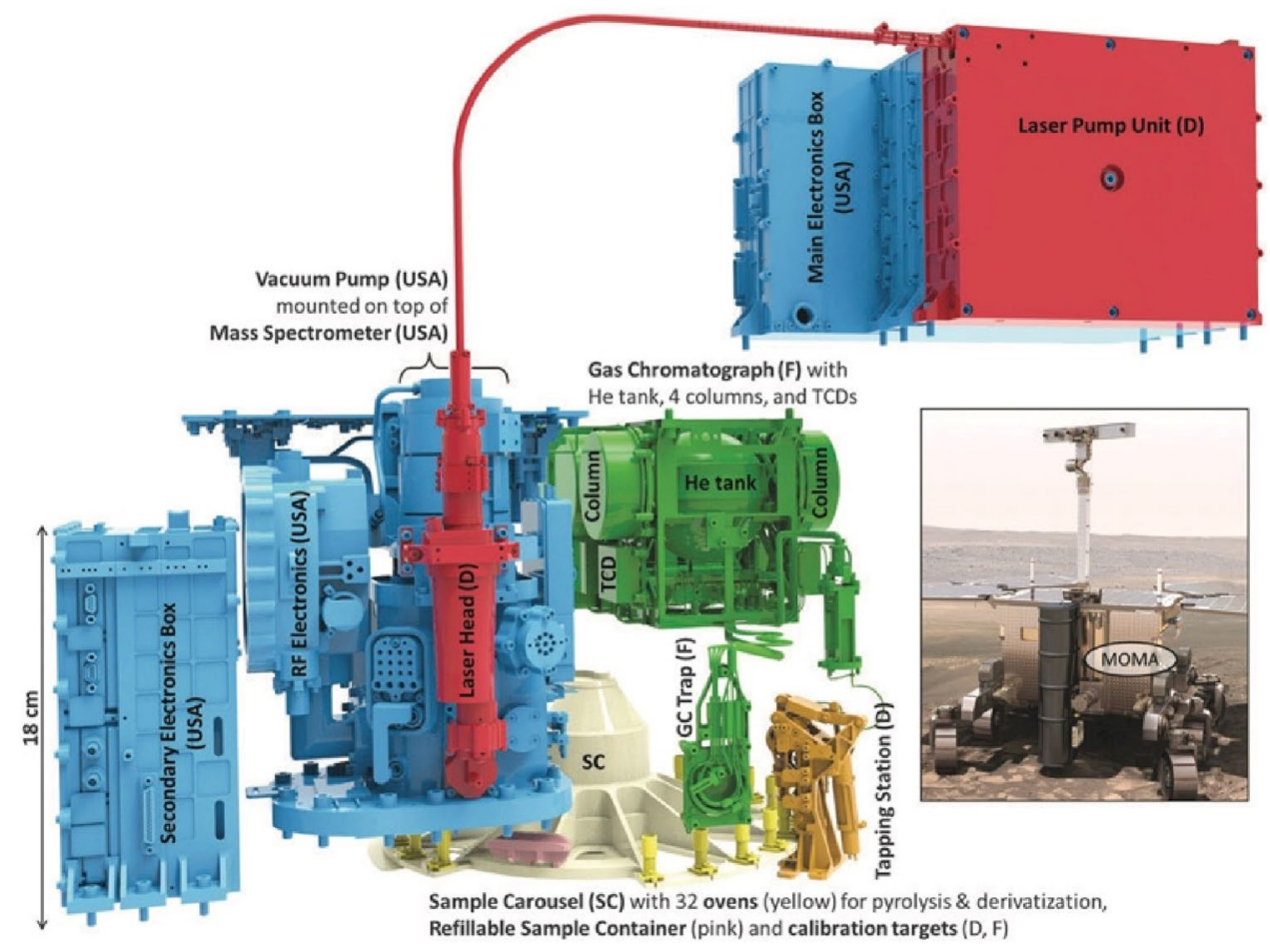

laboratories. Perhaps, they may even foreshadow an era of increasingly autonomous sample collection and testing here on Earth.

\section{The Case for Portable LC}

As analytical instruments reduce in size, they are becoming ever more capable and versatile. Techniques, traditionally requiring larger systems and infrastructure, such as highperformance liquid chromatography and mass spectrometry (MS) are no exception. The pursuit of portable LC has a long history with roots in the early 1980 s and their capability continues to advance.

Before we explore the field of portable LC, we must be aware of where it sits in the wider ecosystem of analytical testing and develop a framework by which to assess the various instruments. Laboratory based systems, unfettered by portability considerations, are technologically the most capable, so analytically running samples on these instruments would seem to be the preferred choice. We are all aware how substantial the footprint of an LC system is and how it is restricted to laboratory benches. Perhaps, the most straightforward approach is to recognise that these systems are not hand portable but can be loaded in their entirety onto specialised response vehicles; the most well-known of these being the Agilent Mobile Laboratory, not too conceptually dissimilar to MOMA. The associated costs, though, prohibit deployment outside emergency incidents to sites to detect chemical and biological warfare agents. The vast majority of field samples are routinely transported to centralised analytical laboratories. By doing so, there invariably exists a disconnect in both time and location between sampling and analysis [22], that has several important consequences with which to contend: (1) samples may transmute or degrade; (2) delays due to logistic turn-around times mean a delay in response or action.

Direct to consumer genetic testing have exposed the wider public to effective field sampling. Many became available in the late 2000s as novelty genealogical services but more recently are able to provide FDA-approved genetic health-risk reports, such as for BRCA mutations, pharmacogenetics of selected variants, and late-onset Alzheimer's [23-27]. Consumers receive a saliva collection kit and post the sample to a laboratory for sequencing. DNA is a robust macromolecule which permits this form of sampling but, in general, liquid sampling and handling of less stable analytes and matrices is particularly challenging in the field. A variety of strategies have been developed including dried blood spot (DBS) testing, swab testing, and lateral flow immunoassays. For instance, DBS sampling is a method that offers logistical advantages when extending the reach of medical surveillance programs, particularly to hard-to-reach populations. Blood samples, such as heel or finger-pricks, are dried onto cellulose paper for storage and transport [28-30]. Once in a laboratory, DBS cards are processed, and the eluate is analysed with conventional instrumentation. As with saliva sampling for consumer genetic testing, these methods are compatible with population-level screening programs due 
to their ease of transportation and storage. While, delays between sampling and analysis will inevitably remain, an important development toward utilising the 'dead' time while clinical samples are in transit was recently reported. Nanthasurasak et al. [31] developed an electrokinetic platform for extracting small-molecule pharmaceuticals from DBS (Fig. 6). This approach is anticipated to not only streamline laboratory workflows once samples are received but may also have potential in limiting sample degradation by limiting matrix interference toward target analytes.

There are applications where sampling and testing onsite is the only route forward, such as those that are extremely time-sensitive or where removal of material from a site is prohibited for safety or regulatory reasons. Traces of bodily fluids, for instance, discovered during forensic investigations at crime scenes are of the utmost importance in order to identify suspects and potentially vindicate innocent parties [32]. Traditionally, both LC and GC are chosen for this type of analysis. Besides body fluid traces, chromatography has also found use in the detection of illicit drugs and explosives [32-40]. However, the requirement to transfer samples and wait for results imposed by traditional LC impedes the efforts of law enforcement agencies. Sample degradation and limited sample size are also restricting factors within the forensic field.

One ongoing research focus is monitoring the levels of herbicides, pesticides, and fungicides and their persistence in the environment following their application [41-48]. Environmental and agricultural analyses require dedicated analytical laboratories for sample testing. It is evident that tools to test factory waste would be ideal in order to minimise the contamination of nearby natural water sources. A portable device capable of making relevant measurements would be invaluable to bodies such as the Department for Environment, Food and Rural Affairs in the UK. Beyond regulation, field testing of wastewater samples would accelerate the implementation of preventative measures to reduce the environmental and human impact of residual toxins.

While the field has yet to fully mature, there is a growing list of compelling applications for portable LC. As sectors become more consumer-focussed, the need for convenient and routine testing will expand. What is likely to help the translation of portable LC out of laboratories is the existing wealth of applications. Portable LC is a disruptive innovator and as such can substantially change industries over time and new applications will certainly open up on-site testing within a variety of new sectors.

\section{Development Considerations for Portable LC}

Suitability for field-portability is not a one-dimensional property and encompasses a wide range of often nearorthogonal considerations. Here we discuss the major considerations and ideal scenarios, then propose a framework for their objective assessment.

For a system to be portable, it must be able to be easily carried or moved. This can, of course, be very subjective but objective frameworks exist: Health and Safety Executive of the UK sets out a number of helpful guidelines for safe lifting capacity [49], depending on the task at hand and posture of the individual. We can define a portable system as that being ideally weighing less than $3 \mathrm{~kg}$ but no more than $5 \mathrm{~kg}$. It follows that the instrument should be a monolithic system, integrating all the necessary computers and electronics into a single housing. We would also contend that this weight limit should include on-board solvent and consumable reservoirs at maximum capacity.

For field use, systems are likely be battery operated but should be able to be recharged in the field. In remote regions, petrol-generator or solar charging is likely to be key
Fig. 6 Illustration depicting intransit DBS sample preparation. While in transit, the analytes are electrokinetically extracted from the membrane powered by two $12 \mathrm{~V}$ batteries connected prior to mailing. On arrival in the lab, manual processing of the sample is significantly reduced, and quantification is achieved rapidly. Reproduced from Nanthasurasak et al. [31]

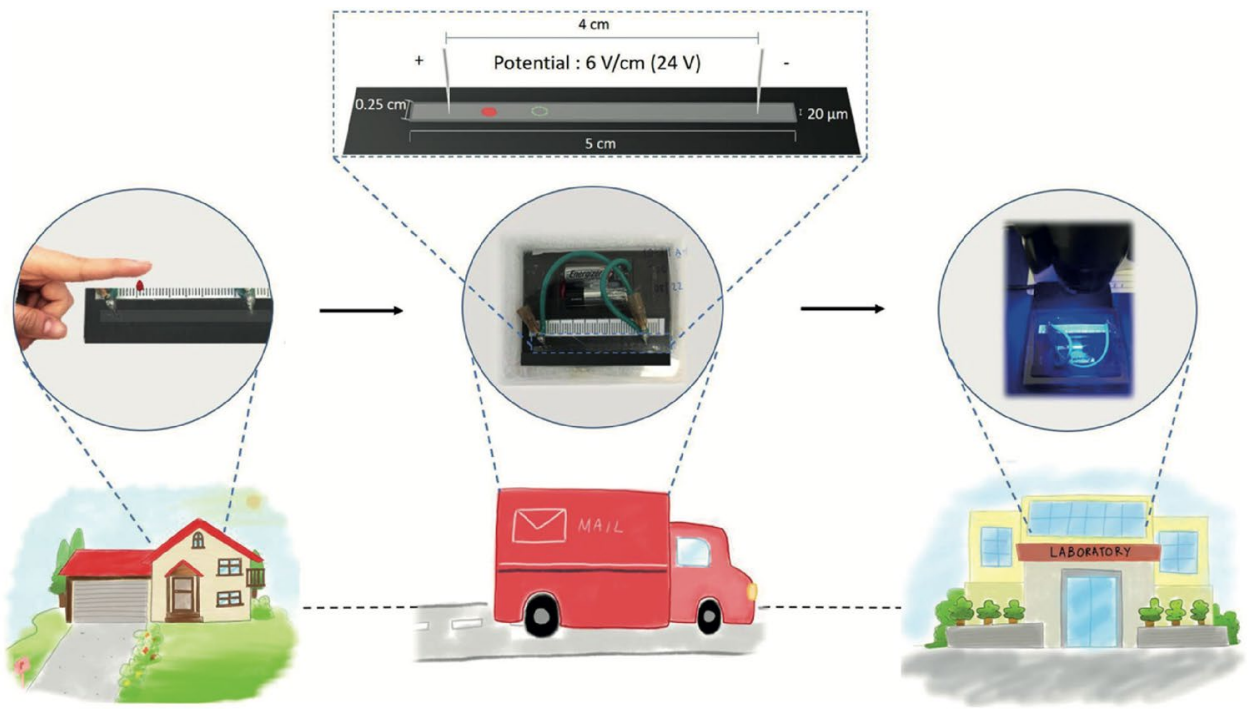


in extending the operation time of the device when access to infrastructure is limited or even non-existent. Researchers should also be aware of other forms of renewable energy generation that can be deployed for ultra-remote settings. They must be capable of operating in a broad range of environments, as measured by their ability to withstand extremes in temperature and humidity as well as resistance to particulates such as dust. Instruments must also be designed to be rugged and capable of withstanding a variety of mechanical shocks while in operation.

We believe one of the most important considerations for portable LC is cost. The growing need for field-based or point-of-care measurements is best supported by miniaturised systems that not only maintain or exceed the typical performance of their laboratory-based counterparts but to do so in an affordable and therefore accessible manner. Arguably, at a minimum, the cost per test/run in the field should strive to be no more than, or competitive with, returning samples back to the laboratory. Depending on turn-around time, HPLC tests in centralised laboratories are on the order of $\$ 10-\$ 100$ s US. For perspective, the cost of on-site analysis per test using colorimetric test strips or more capable paper microfluidics is on the order of $\$ 0.01$ US or less. Pioneering approaches that adopt rapid and additive manufacturing, such as $3 \mathrm{D}$ printing, are likely to become critical to reducing costs in successive generations of portable LC [50-52].

Another criterion in the framework, important for widespread adoption and validation of platforms, is how open the hardware, software and protocols are to the community. Foundations, such as the Open Source Hardware Association (OSHWA) have published a well-defined standard for open-source compliance, which aims to foster technological knowledge and encourage research that is accessible and collaborative. Given the commercial potential of these systems, players may take some convincing. However, the prevalence of prototype instrumentation being reported that themselves incorporate open source components, e.g. single board computers, ought to be sufficient encouragement to adopt open initiatives, as much as possible.

An often overlooked or underestimated aspect to field chemistry is the unintended environmental impact it may have on ecosystems. Primarily, LC method development is focussed on precision, accuracy and repeatability. Greener practise is growing in laboratories seeking to reduce their environmental burden, of which large amounts of chemical waste often represent an important part. Encouragingly, while portability can make some criteria harder to achieve, miniaturised portable systems typically require dramatically lower solvent consumption. Welch et al. [53] recognised that while portable analytical instruments are breaking free of the traditional boundaries of the laboratory, the dependence of techniques on regulated organic solvents remain. They investigated the use of liquids readily available in supermarkets, such as spirits, as mobile phases and additives for fieldbased LC and liquid chromatography-mass spectrometry (LC-MS). Green solvent replacements that can be disposed in ordinary waste or recycling streams should be actively pursued and their performance reported alongside more conventional methods (Fig. 7). Given the supply chain for unregulated liquids is naturally more ubiquitous, this will, where possible, have the added benefit of prolonging deployment times without the need to return to centralised facilities to replenish such consumables and help drive down costs. A simple and efficient approach for profiling the greenness of HPLC methods has been put forward by Gaber et al. [54] To aid researchers, they developed a software based environment assessment tool (HPLC-EAT) to score and rank the greenness of methods in an accurate and logical way. Therefore, we encourage the use of green solvent replacements for field applications.

Chromatographic performance is both the single most important question regarding any chromatographic system, and also the hardest to define, particularly as it is typically dependent on the methods being employed. The performance of conventional LC instruments has improved through microbore and capillary columns, the chemistry of the stationary phase, and, of course, the pumps that can overcome the increased back pressures demanded by these advanced methods. Detection strategies such as multispectral detectors and hyphenated mass-spectrometry techniques have been critical in the adoption of LC. Given the multitude of factors that contribute to an LCs performance, it is the trickiest element to reduce to simple commensurate parameters. It is, perhaps, even detrimental. It should be expected that portable LC would have the requisite accuracy, precision and repeatability that any application requires. The field can run the risk of engaging in a technological arms race and forget the underlying principle of what it is trying to achieve-delivering HPLC anywhere it is needed through innovation.

Frequently the requirements for LC portability we have outlined above are discussed in the literature [55, 56], yet there is currently no general framework to allow comparison of advances in the field, and reporting is often not consistent/commensurate the in primary literature. To overcome this, here we propose a set of criteria to assess a system's portability/field-suitability. The BETTER (portaBle fiEld Testing sTandard framEwoRk) criteria 2020 (Table 1) proposed here are intended to act as a framework to facilitate more objective comparison, encourage better reporting, and act as a development framework for the community. BETTER 2020 endeavours to account for non-chromatographic characteristics (e.g. costs, robustness), along with oftenneglected requirements with considerable impact on a system's field-applicability (portability, solvent requirements, etc.). Levels are often defined by usability: for example, it 


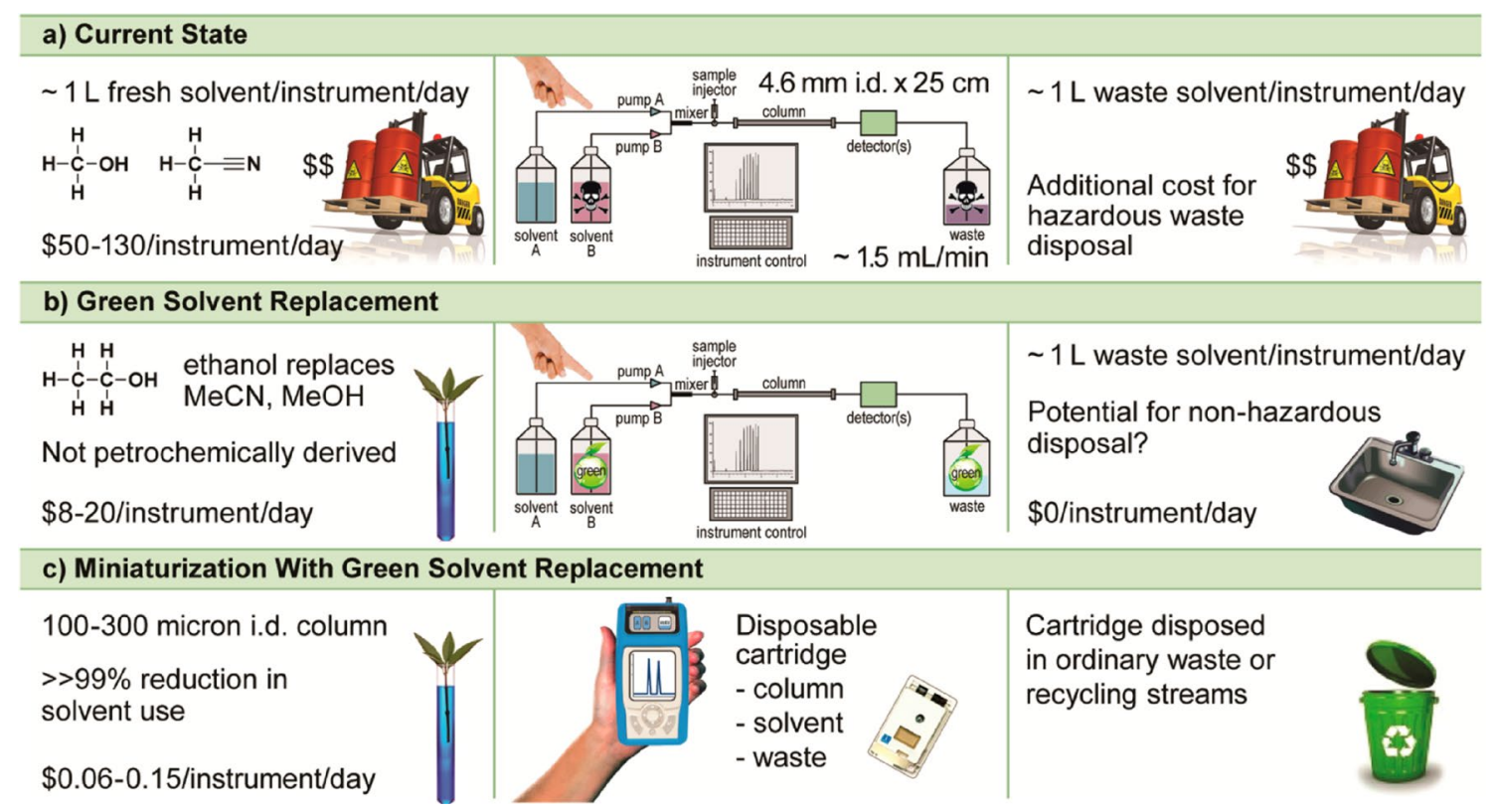

Fig. 7 The current state and future alternatives for environmentally friendly HPLC analysis in which a existing procedure involves high flow rates of approx. $1.5 \mathrm{~mL} / \mathrm{min}$ with large bore columns $(4.6 \mathrm{~mm}$ I.D. $\times 25 \mathrm{~cm}$ ) producing and consuming over $1 \mathrm{~L}$ of fresh and waste solvent daily per instrument. b Switching to greener solvents such as distilled ethanol available from local markets reduces the running

is more relevant to a user whether a system can realistically be carried on a scrambler bike or by a hiker, than any more specific variable. BETTER grade levels (each ranging from 1 to 5) are selected both to represent the current range of developments, and community goals. To date, portable LC systems meet some of the grade 2 and 3 criteria. The grade 4 and 5 criteria, then, may be seen as a challenge to be met by next generation devices; indeed, no instrument reported to date reaches Grade 5 in any category (see SI). While currently stretching to Grade 5, The BETTER criteria 2020 are designed to be open-ended. We intend to improve the framework, as technology advances, and capturing interaction with the community.

In our review of portable LC technology, we endeavour to assess previously reported instruments using the BETTER criteria 2020 (see below, and SI for details). Crucially, these ratings are intended to assess the whole system required to perform chromatography. In estimating ratings, we note that many factors are not addressed in original publications. These "externalities" often include: solvent reservoirs; computer/controller; power source; and consumables. For example, in the case of a miniature instrument which weighs $250 \mathrm{~g}$, but which requires a robust power supply to operate in the field, the weight should not be assessed purely on the basis of the instrument, but the whole system. Lacking the relevant data, and to allow objective comparison, we would estimate this by adding the weight of a petrol generator and costs and reduces the need for disposal of hazardous waste solvents. c The trend towards shorter narrow-bore columns lowers solvent consumption and waste generation to a few millilitres per instrument per day with the potential to encapsulate within a handheld platform. Reproduced from Welch et al. [53]

fuel required to maintain the instrument in operation for the stated "operation time away from the laboratory", along with the mass of solvent required. Our standard approach to these calculations is listed in the SI. We have used information that is available in the relevant academic publications and where information is missing or not reported we have endeavoured to make assumptions in good faith. A public GitHub repository of the current BETTER standards, and notes on assessment, will be maintained to make this a sustainable endeavour, as can be seen at https://better-hplc.githu b.io. Additionally, we encourage the submission of accurate and complete data to the repository.

Importantly, we must note that these ratings are not intended for addition to produce an overall score: the importance of different attributes and the practicality of a system depends heavily on the requirements of the intended application, and the degree of flexibility required. As such, more BETTER is not always best, and we eschew any attempt to further simplify to a single numerical assessment.

\section{The Components of a Portable LC}

High performance liquid chromatography (HPLC) instrumentation are typically made up of four main componentsan injection system to introduce the sample; an eluent pump to generate a specific flow rate; an analytical column which 


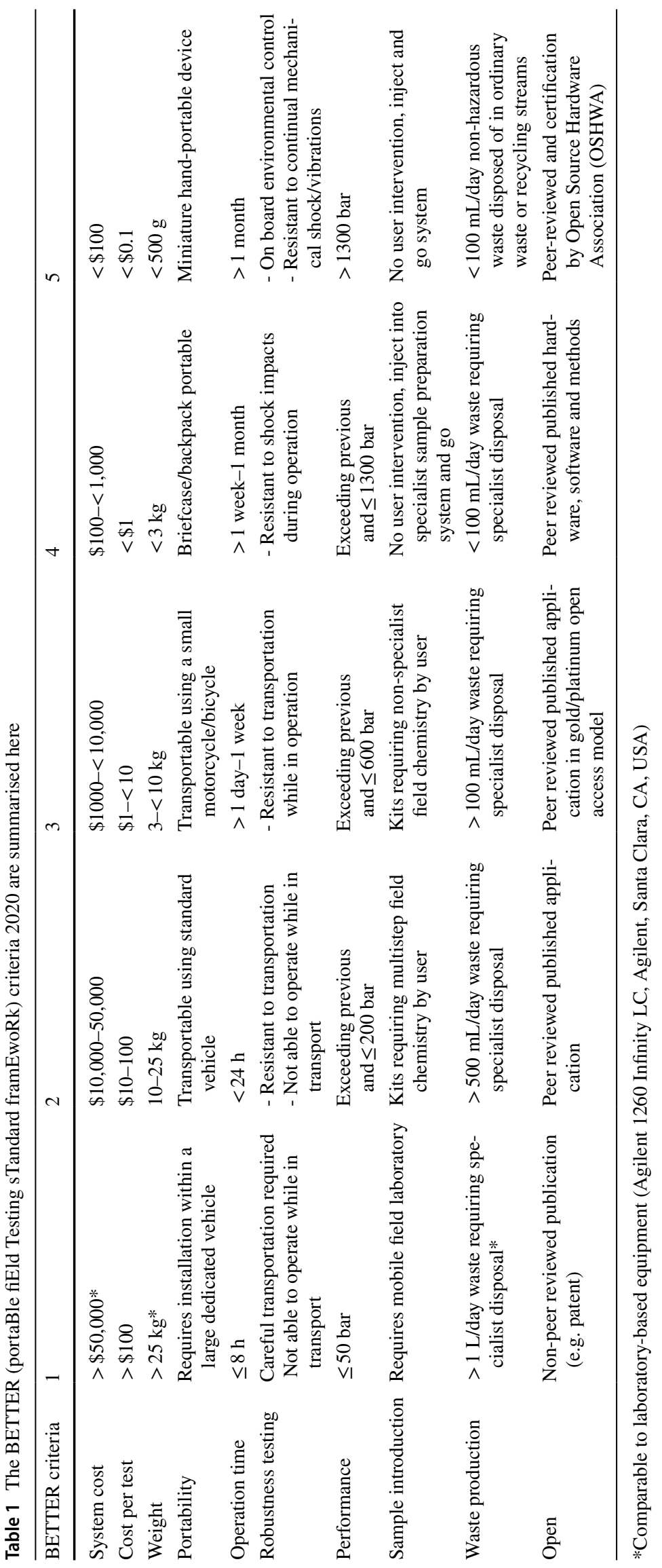


separates the analytes within the sample based on physical and chemical parameters; and a detector to identify and quantify the analytes as they elute. The driving force behind portable HPLC instruments has been the miniaturisation of these components, the idea being to reduce the physical dimensions and weight while ensuring that all elements retain their analytical capability. This introduces increased cost and complexity in manufacturing and has potential impacts upon reliability and consistency.

\section{Injector}

Injection assemblies have not changed significantly for portable liquid chromatography (LC) instrumentation. Injectors are inherently small and are designed to introduce samples with high precision and minimal flow disturbance. They are critical to reproducibility and so it is understandable researchers have relied on tested designs. However, they are expensive components and LC systems can incorporate one of two injectors depending on whether the mobile phase (MP) flow is halted during injection. For stop-flow injectors, the sample is introduced while the MP flow is stopped, whereas continuous-flow injectors do not disrupt the flow. Both systems require narrow tubing connections and low dead volumes to reduce band broadening and peak tailing, respectively. It is worth noting that stop-flow injectors provide significantly lower dead-volumes and are, therefore, being found in a large number of prototypes in recent years [57-59]. Six-port valve injection systems are widely used in LC systems to introduce the sample without interrupting the flow of MP. By rotating the valve, either manually or using an automated system, the sample loop is interfaced with the high-pressure MP and the sample flows to the column via the pump. These automated setups immensely increase the ease-of-use of the system since operator input is minimised but do so at considerable cost.

Microfluidic injection systems that mimic the operation of standard six-port valves have been reported [60, 61]. These are traditionally based on the deformation of a flexible poly(dimethylsiloxane) (PDMS) membrane to provide a means of flow control [62]. Integrating on-chip valving can help to avoid large dead volumes and external actuating components. However, they predominantly focus on biologically compatible, low-pressure applications. High pressure variants have been reported [63]. For example, Sharma et al. [64] developed a microvalve able to withstand back pressures of $20 \mathrm{MPa}$, limited by the rigidity and fluid connections of their device (Fig. 8). Although, only tested with air and water, the only materials in contact with liquid are parylene and stainless steel and so should afford it good chemical resistance to aggressive solvents. Sample injection and switching valves for LC are incredibly reliable and so newer classes of microvalve will need to be proven to offer similar performance over extended use.

\section{The High Pressure Pump}

The miniaturisation of LC pumps is critical to successful portable LC equipment. Encouragingly, this a very active area of research with several very different approaches. Miniature pressure-generating pumps based on piston pumps; syringe pumps; latent energy pumping; and electroosmotic pumps (EOPs) have all been reported [58, 65-67]. There are certain features which the ideal portable pumping system should possess: it should be lightweight and batteryoperable; capable of a wide range of flow rates $(\mathrm{nL}-\mu \mathrm{L}-\mathrm{mL} /$ min); provide high flow rate accuracy and precision; generate a constant flow rate independent of back pressure; capable of gradient elution with high reproducibility; simple to

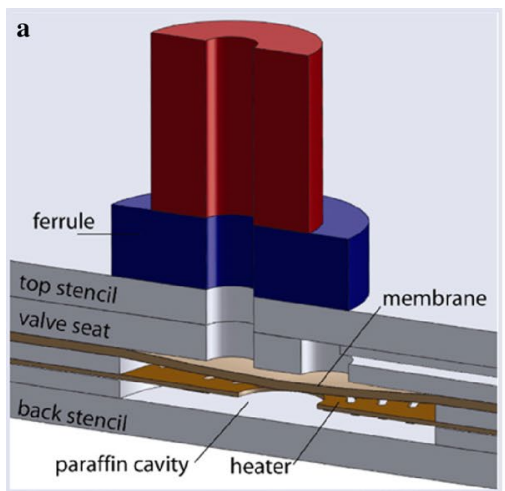

Fig. 8 a Schematic of a thermohydraulic actuator activated by the high hydrostatic pressure of paraffin wax upon melting. The volume decrease experienced on solidifying the paraffin is compensated by a membrane deflection from the valve seat resulting in an open fluidic

b

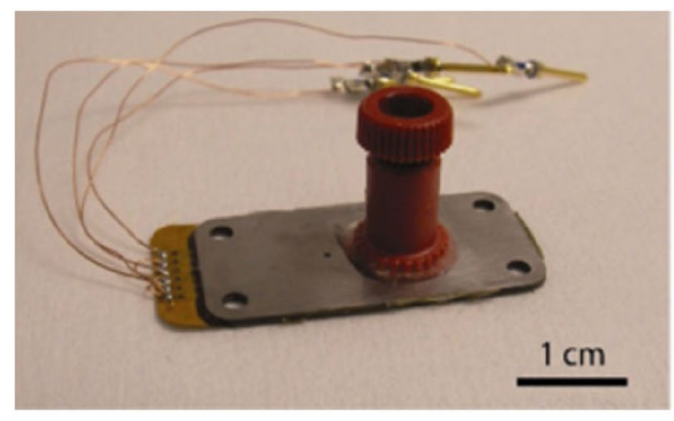

channel from the inlet to the outlet. b Image of a nanoport microfluidic interface attached to the microvalve using epoxy glue. Reproduced from Sharma et al. [64] 
operate and repair when needed; and produce minimal pulsation noise [56]. While achieving a pumping mechanism incorporating all of the above capabilities is a challenging venture, this must also be delivered in a portable form-factor.

Solvent reservoirs are either upstream of the pump, and at atmospheric/low pressure, or downstream, and at high pressure. This has a few critical consequences to the design and performance of a system overall and how it is operated in the field. Solvent reservoirs upstream of the pump may be replenished during runs and needn't be of a special construction e.g. piston pumps that are multi-stroke systems and draw solvent from glass vials. Pumps that require the solvent reservoir to be downstream are typically single stroke systems e.g. syringe pumps driving mobile phase from a syringe. In this case, the reservoir of solvent dictates the maximum operating time before analyses must stop and requires replenishing. Due to the reciprocal relationship between the volume of these reservoirs and the pump pressures that can be achieved, achieving higher and higher pressures, a syringe pump inevitably tends to the characteristics of a piston pump that is limited to a single stroke. Inevitably, a trade-off must be struck.

Piston pumps are the predominant form of pump in modern LC systems. The main disadvantage of these pumping systems is pulsatile flow due to the non-continuous manner in which the liquid is delivered. This class of pumps comprise multiple moving parts which require careful maintenance and upkeep. Well maintained LC pumps are known for their longevity; however, it is not clear how robust they are to field use over an extended time. In the early days of LC, syringe type pumps were commonly used in driving solvents, particularly as part of isocratic methods. Syringe pumps comprise a stepper motor which drives the plunger of a syringe filled with solvent along worm gears. The major drawback of syringes are their finite volume which necessitates continual refilling and can be problematic if the separation is not completed before the syringe volume is consumed, particularly if samples are precious.

Syringe and piston type pumps are similar in the type of flow they generate. Characteristic fluctuations or oscillations in flow rate originate from their mechanical operation. For piston pumps, pressure pulses arise due to the action of the piston. The waveform of these pressure oscillations may be flattened by modifications in the design of the cam or modifying the speed of the motor during the intake and delivery strokes [68]. However, pulses are inevitable and pulse dampeners are used to reduce fluctuations further.

Pressure-driven pumps control flow rate by applying a pressure to a sealed reservoir. The pressure applied to the fluid, drives it through the system. Latent energy pumping and pneumatic pumping systems represent a branch of mechanisms that use the force of compressed gases to generate mechanical effects. Due to the high precision with which gas may be regulated, pulseless flows with incredibly high stability may be achieved. High pressures of several hundred bar may be achieved using compressed gases and with pneumatic amplification pressures of several thousand bar are possible. A major advantage of pressure-driven pumps is their compatibility with a wide range of solvent reservoir volumes. Pressure-driven pumps are not unlike syringe pumps in that they can be considered to apply force upstream of the solvent reservoir. While this does mean analyses must be stopped as the solvent reservoir is replenished, the compatibility with large reservoir volumes means this is not a major issue in practical terms. One of the major factors limiting portable LC is the overall electrical energy budget of the system. Pressure-driven pumps incorporating pre-pressurised sources of gas are capable of significantly reducing, or removing entirely, the electrical energy cost of the pump and so are attractive toward enabling extended field operation.

Metering flow rates for pressure driven flow requires a flow rate sensor and feedback control; whereas for syringe/ piston pumps, the flow rate may be easily controlled by setting the speed of the motor. However, it is important to note that flow rate sensors are still necessary during the transient period as flow rate is altered. The responsiveness of piston and syringe pumps for high pressure applications can be slow and is measured on the order of tens of seconds to minutes. Pressure-driven pumps are well known for their responsiveness and are measured on the order of milliseconds to seconds.

The field of microfluidics and $\mu$ TAS (micro total analysis systems) has driven advances in other pumping systems such as EOPs [69, 70]. Alike pressure-driven systems, EOPs are capable of generating pulse-free flow and have no moving parts. By way of their operation, they are inherently capable of sensing the magnitude and direction of flow without the need for additional sensors [71]. Back pressures are overcome by the phenomenon of electroosmosis in which bulk liquid flow is produced by applying an electric field across a porous dielectric media (Fig. 9) [69]. The major benefit is that the pumps are readily miniaturised and do not involve any moving parts.

There are concerns over the reliability and compatibility of EOPs for HPLC. For example, while both polar and, recently, non-polar solvents may be used-as seen within non-aqueous capillary electrophoresis [72-77], and the flow generated can change due to the adsorption of analytes from the sample or sample matrix onto the elements of the pump. Although, this can be avoided by isolating the pump from the downstream fluidics, it has the effect of constraining EOPs from being a continuous pumping system to one limited by a solvent reservoir akin to a syringe pump. The main drawback of EOPs lies in how they are powered. They are required to be operated under high electric fields which 


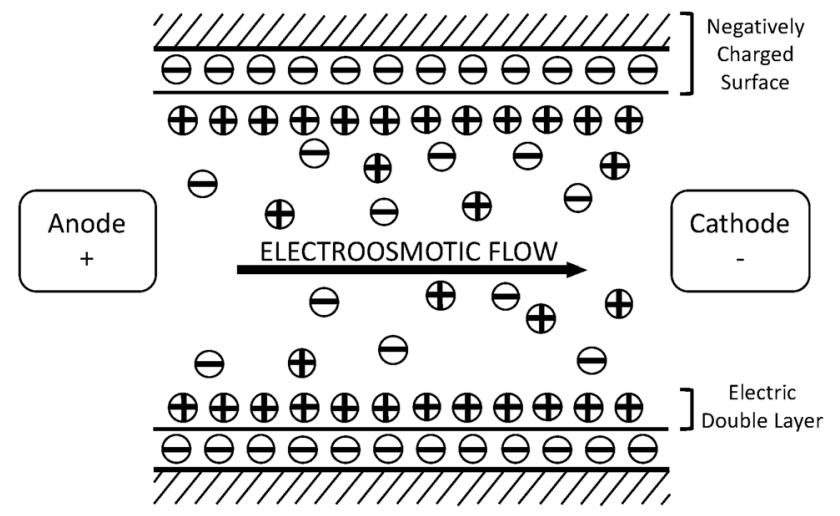

Fig. 9 Schematic of the electrical double layer and electroosmotic flow generated by the application of voltage at the terminals of the capillary. The electric field generated forces an excess of cations in the diffuse layer. This, in turn, forces a movement of the diffuse layer towards the cathode, creating a flow of the bulk solution in the same direction

results in bubble formation and variations in $\mathrm{pH}$ as a result of electrolysis at the electrodes [22]. The development of alternating current-powered (AC) EOPs address this but the necessity of bulky power supplies which are not easily implemented into portable systems remains.

Gradient elution LC systems are usually developed in one of two ways: (1) Multiple pumps are combined each tasked with driving a proportional amount of a different solvent, a common example would be binary pumping; and (2) single pumps driving a plug of fluid pre-mixed upstream. Only binary and electroosmotic pumps are compatible with either approach. Whereas, syringe and pressure-driven pumps may only achieve gradient elution by driving mobile phase that is mixed downstream but before entering the analytical system.

\section{Column Technologies}

Column miniaturisation has had a significant impact on the development of portable LC. Column dimensions, the size and chemical properties of packing materials, and in particular internal diameters (I.D.), have progressively reduced. This has decreased the solvent consumption and required sample volumes, and increased the detector sensitivity. By decreasing the I.D. of the column, the eddy diffusion term of the Van Deemter equation is reduced since there are less paths through the column; therefore, there should be less opportunity for band broadening to occur. Conventional columns ( $4.6 \mathrm{~mm}$ I.D.) were superseded by microcolumns (0.5-1.5 mm I.D.) followed by capillary columns (0.01-0.5 mm I.D.), and LC miniaturisation has improved as a result. Decreased solvent consumption enables lower volume solvent and waste reservoirs and subsequently a more compact and portable LC. On the other hand, decreasing column I.D. has been challenging for portable pumping systems to counteract the rise in backpressures.

The tendency to decrease particle size has led to very high efficiency columns. While this can improve the resolution and separation ability, LC systems can suffer from high backpressures currently unattainable by miniaturised pumps. The advent of silica monolith columns provided a solution in which low backpressures and high efficiency columns were the new trend.

Within the past two decades, the field of microfluidics has excelled in realising novel and unique platforms for studying chemical and cell-based analyses. So too have advances in LC column on chip technology; microfluidic alternatives to tubular columns have been presented in the form of planar geometries comprising micro-pillar arrays. These micro-pillar array columns ( $\mu$ PACs) are fabricated via a lithographic etching process onto a silicon chip creating a completely ordered separation bed (Fig. 10a). $\mu$ PACs benefit from high permeability and low dispersion due to the structured framework. Also, lower backpressures are possible resulting from the freestanding character of the pillars. These inherent properties generate the potential for LC performance with high resolution and sensitivity [78].

In recent years, the opportunity to drastically improve the performance of chromatographic separations beyond what is currently possible has been realised. The advance of 3D printing technologies present a newfound ability to fabricate precisely ordered stationary phases of varying shapes which have the potential to increase the efficiency of chromatographic columns [80,81]. The developments in this research are impressive and may, in the future, improve the analytical sector significantly.

\section{The Detector}

Effective detection systems have been the focus of numerous research groups for decades. A considerable number of research articles and reviews have directed their attention to the potential of developing mobile and handheld devices based on various detection methods. Some of these include Raman [82-87], ultraviolet (UV) [88], visible [89], ultraviolet-visible (UV-vis) [90-92], near-infrared (NIR) [93, 94], Fourier-transform infrared spectroscopy (FTIR) [95, 96], induced fluorescence [97-99], electrochemical detectors (ECDs), and X-ray fluorescence [100-102]. The vast majority of these methods can be adapted to detect and quantify eluting analytes in an LC instrument. Thus far, however, absorbance detectors have found common use in portable HPLC systems mainly owing to the ease of miniaturisation and integration.

Moreover, the coupling of portable LC to a variety of detection methods provides the opportunity to develop a $\mu$ TAS. This would be achieved with a portable system which 


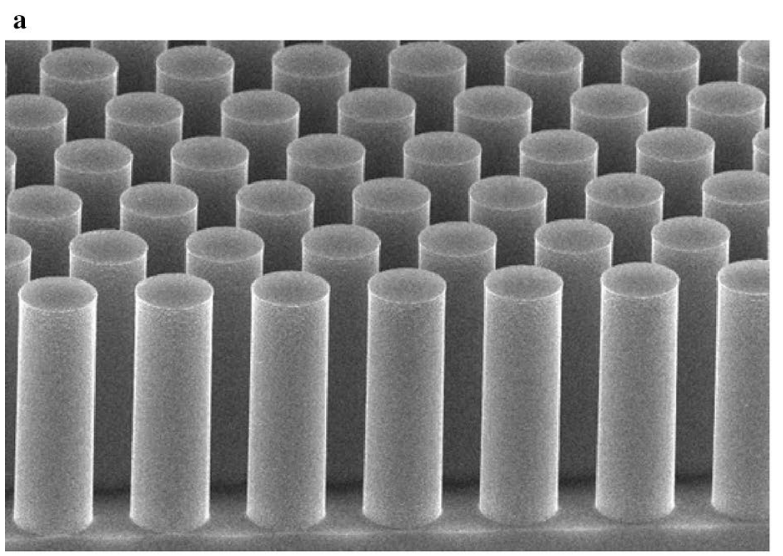

b

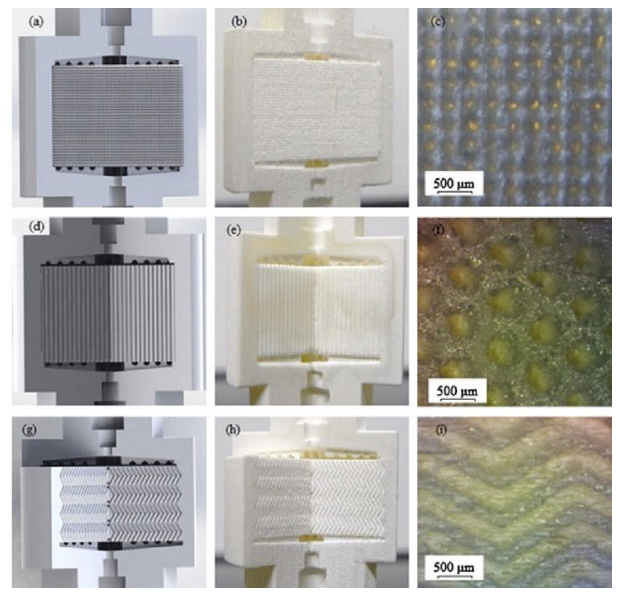

Fig. 10 a Scanning electron microscope image of the micro-pillar arrays in $\mu \mathrm{PAC}$ devices. b Porous beds with three geometric designs: (a)-(c) simple cubic arrangement of beads, (d)-(f) parallel channels of a monolith, and (g)-(i) herringbone-shaped channels. The first column shows the CAD designs, panels (a), (d) and (g), while the

is able to perform the entire analytical process from sample handling to electronic output of results at the point of sampling in a smaller footprint compared to a conventional system [103].

Quantitative analysis is traditionally achieved using ultraviolet-visible (UV-Vis) spectrophotometry methods. In conventional systems, broadband spectra are delivered by lamps. Given their fragility, long warm-up times and demanding power requirements, these have limited applicability in a field setting. Instead, systems have exclusively adopted light-emitting diodes as light sources.

Previously, the sensitivity and limits of detection (LOD) of UV-Vis detectors were improved by increasing the path length of the sample along the detection path. However, in applications where the elution volume is low, the I.D. of the flow cell should be reduced accordingly. The consequence of this is the increased noise levels resulting from the decreased optical transmittance. The advent of Z-shaped flow cells (Z-cell), see Fig. 11a, decoupled the dependence second and third columns display the printed models, panels (b), (e) and (h), and 20x magnification of the channels, panels (c), (f) and (i), respectively. Reproduced from Pharmafluidics [79]. Reproduced from Fee et al. [80]

of the LOD to the width of the flow cell. Chervet et al. [104] experienced a 14 fold signal increase resulting from a theoretically 80 fold path length increase. The resultant sacrifice in time resolution can result in peak broadening; however, this occurs on a millisecond to second timescale which would only have minimal impacts on anything other than fast ultra-high performance liquid chromatography (UHPLC) instruments. In addition, the backpressures that can be achieved are restricted by the glass windows of the Z-cell. However, given that the pressures achieved by the pumping mechanisms in portable LC's often fall below commercial HPLC or UHPLC systems, this issue can be easily overcome using a pressure regulator. This highlights an important question which is often overlooked when selecting appropriate detectors as well as other components; are the analytical capabilities sufficient for the intended purpose? As such, components considered unsuitable for benchtop HPLC instruments may perhaps find use in portable LC devices.
Fig. 11 a Schematic of

(A) Z-shaped flow cell and (B) standard flow cell. The increased optical path length of the Z-cell is indicated by the double headed arrow. $\mathbf{b}$ Schematic of a total internal reflectance (or light-pipe) UV detector cell, as used in UHPLC applications a

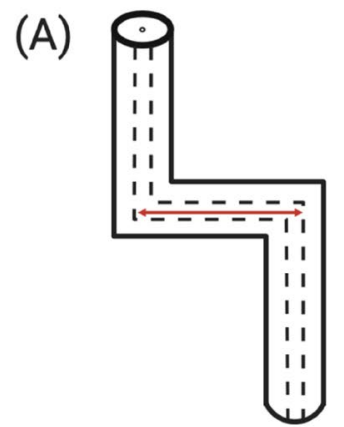

b

(B)

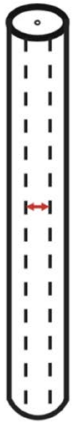

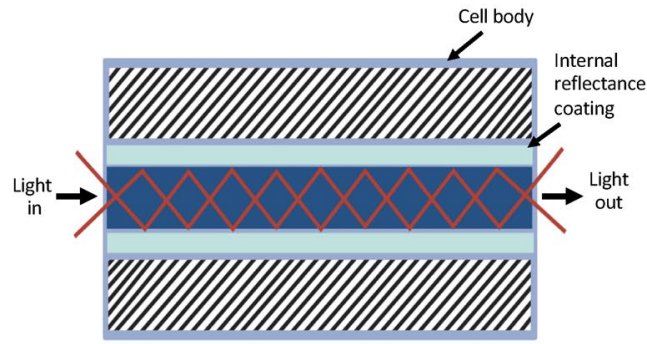


With the introduction of UHPLC systems, peak broadening due to high volume flow cells became a more pressing issue. Reduction of the cell I.D. was necessary to overcome this issue; however, by doing so, the amplified effect of refractive index brought about another issue of light scattering. The solution to this was achieved with the design of the total internal reflectance (TIR) cell (Fig. 11b). The cell walls are coated with a reflective material such that any light that hits the walls of the flow cell is reflected along the walls several times before reaching the other end. TIR takes the concept of the Z-cell a step further and significantly increases the path length of the cell enabling the use of longer flow cells with better sensitivity and reducing peak broadening in comparison to shorter length and larger I.D. flow cells [105]. While TIR cells may appear to be the onestop solution for UV-Vis detection, uncertainties remain with respect to their fragility. Reports have emerged of damage to the cell coating once the cell has dried out and then exposed to UV light [106]. Although certain safeguards can be put into place to prevent this event occurring, the robustness of the cell remains a concern. This leads to a more nuanced point regarding detection systems, how does one design a system based on current detection methods that is robust and stable in the field. By addressing this, as well as prior and subsequent points, the LC community will better accomplish a portable LC system relevant to the needs of field-researchers.

Thus far, the UV-Vis detection methods discussed have been examples of end-column detection. In recent years, on-column methods have become the preferred method for capillary columns. This is mainly due to the elimination of additional band-broadening from tubes connecting the column and detector, ultimately, preserving the separation efficiency [58]. It should be taken into consideration that for effective on-column detection, accurate and narrow focusing of the light beam into the capillary is essential. In addition, slits are used to eliminate stray light. However, this reduces light throughput and can, as a result, reduce the signal-to-noise ratio (SNR) of the detector [107]. For a portable system operating in the field where shocks and vibrations are commonplace, sensitive optical arrangements are likely to be quite problematic and will require persistent adjustment and troubleshooting. As a result, the previous question is very applicable to on-column detection; how can an on-column detection system be designed such that it is robust in the field.

By definition, a handheld device refers to a highly compact device able to be held with one hand. While, a portable device is one which is adequately light and small to allow it to be easily carried or moved [108]. As mentioned previously, several handheld UV-Vis instruments have been prototyped and successfully made available to the market. The miniaturisation of LC equipment, and more broadly, the evolution of $\mu$ TAS has accelerated the need for miniaturised detectors and in particular UV-Vis. To date, modern applications of these instruments include a novel spectrometer designed to provide measurements of ozone profiles, aerosol optical depth, Volatile Organic Compounds, and other key air quality parameters [109]; a portable, liquid core waveguide photometer used for the analysis and determination of DNA samples and cholesterol, respectively, in human-serum [110]; and most interestingly, an inexpensive 3D-printable smartphone spectrophotometer which can be used to teach students concepts such as the Beer-Lambert Law with appropriate functionality and analytical accuracy [111].

These modern-day applications showcase the on-going potential of spectroscopic detection, and the growing movement for robust handheld and portable equipment. One may identify an underlying trend towards more rugged equipment with the ability to withstand shocks and vibrations common in the field. Consequently, some may argue that there is a direct trade-off between robustness and analytical capacity. However, it is important to consider the type of results needed in outdoor operation. In many cases, researchers desire rapid identification and detection as opposed to highly accurate quantitation. As a result, the question that needs to be addressed is does the ability to conduct confirmation tests in remote locations justify the constraint on analytical accuracy. The likelihood is that until there are further improvements and advances in the detection, the scientific community would greatly benefit from a device which meets the majority of the requirements.

Whether these novel UV-Vis applications are suitable for implementing into a portable LC system is yet to be revealed. Challenges may hinder adequate performance of the UV-Vis detector, namely the requirement for a chromophore within the analyte sample to allow light absorption, and the sensitivity of the detector to bubbles-in particular, the issue of outgassing. Limitations of absorption detection arises from the need for the analyte to absorb in the ultraviolet or visible region. While derivatisation steps can be introduced for analytes which do not absorb in these regions, this can be difficult for point-of-sampling analysis in the field.

The developments of miniature mass spectrometers are proceeding quickly and highlight the potential for a miniaturised hybrid instrument based on MS and LC [112-121]. In contrast to UV-Vis, mass spectrometry possesses the ability to provide a significant amount of quantitative and qualitative information free from complex derivatisation protocols. MS serves as a valuable detector since high sensitivity and mass accuracy can be obtained, as well as the added benefit of structural information which is often used for characterisation purposes.

The trend towards miniaturisation of LC components has meant that liquid flows have become more suitable for MS. While other ionisation techniques, such as matrix-assisted 
laser desorption/ionisation (MALDI), are available for MS analysis, electrospray ionisation (ESI) sources are now the method of choice for a variety of hyphenated LC applications due to the ease of analysis of involatile, thermally labile biomolecules [122]. Interfacing LC with MALDI-MS requires offline methods that are labour intensive and may result in sample loss [122]. ESI is an example of a softionisation approach in which molecular ion fragmentation is kept to a minimum. Large, fragile biomolecules and proteins can be analysed with no dissociation and the opportunity to generate multiply charged ions, making ESI a popular ionisation technique within proteomics.

Recently, Zhao et al. successfully interfaced a miniaturised UHPLC instrument with a commercial ESI-MS system and demonstrated the analysis of trypsin-digested bovine serum albumin [123]. The coupling was achieved by fabricating a capillary column with an integrated ESI tip in which one end of the column was melted and made into a narrow tip (approximately $25 \mu \mathrm{m}$ I.D.). Further work focussing on interfacing this miniaturised UHPLC system with miniaturised ESI-MS systems has the potential to revolutionise field-based proteomics research as well as clinical disease diagnosis.

In recent years, the efforts of developing miniature MS systems have been characterised in the form of the 'Mini 12'. Li et al. aimed to develop a proof-of-concept system (Fig. 12) with tandem-MS (MS/MS) capable of direct sample analysis in complex matrices without prior separation or traditional pre-treatment. The benchtop system weighs $25 \mathrm{~kg}$ and has outer case dimensions of $49.8 \times 56.1 \times 41.9 \mathrm{~cm}$. In a similar space, Giannoukos et al. have developed a quadrupole mass analyser based miniature mass spectrometer capable of human volatile organic carbon detection for the investigation of illegal human trafficking in border checkpoints [124, 125]. This field is further summarised in several reviews to which the reader is referred to $[116,126]$.

As previously discussed in "Development Considerations for Portable LC" section, it is important to establish the difference between field portable and field transportable. Field portable refers to systems which can be moved by all users with ease and without necessitating dedicated travel protocols. Whereas, field transportable alludes to benchtop systems which can be readily deployed as field laboratories with great care to avoid damage from impacts and jolts. The miniaturised MS systems currently undergoing development within research and commercial entities resemble field transportable rather than field portable systems.

Great efforts have been made to miniaturise and implement UV-Vis detection systems into portable LC. However, the question that remains is whether this detection technique can provide suitable coverage in comparison with MS and should our attention collectively be directed at miniature MS as the primary detector for in-field LC. This discussion becomes more intriguing as our attention is focused on the typical performance indicators of a detection system: sensitivity and selectivity. The chromatogram provided by UV-Vis detectors are a result of the absorption spectra of compounds excited in the ultraviolet and visible regions. It can be very difficult to differentiate between analytes eluting with similar retention times without incorporating multiwavelength detection. However, with MS and UV-Vis detection used together in a workflow, analyte separation based on molecular weight in real-time enables improved selectivity since it is highly unlikely that a pair of analytes will possess identical UV-Vis spectra and $\mathrm{m} / \mathrm{z}$ ratios. It is worth remembering that traditional MS is impeded by its inability to distinguish between enantiomers of a chiral compound. Chiral analysis can be achieved using a hyphenated technique such as LC-MS. This approach utilises an enantioselective column or a chiral reagent to produce diastereoisomers [127].

As previously mentioned, adequate sensitivity in $\mathrm{UV}-\mathrm{Vis}$ detection requires good chromophores to absorb the light from the source. For those compounds which contain poor chromophores, this detection method becomes more laborious and less useful. On the other hand, MS detection is not hindered by the same requirements, and can therefore,
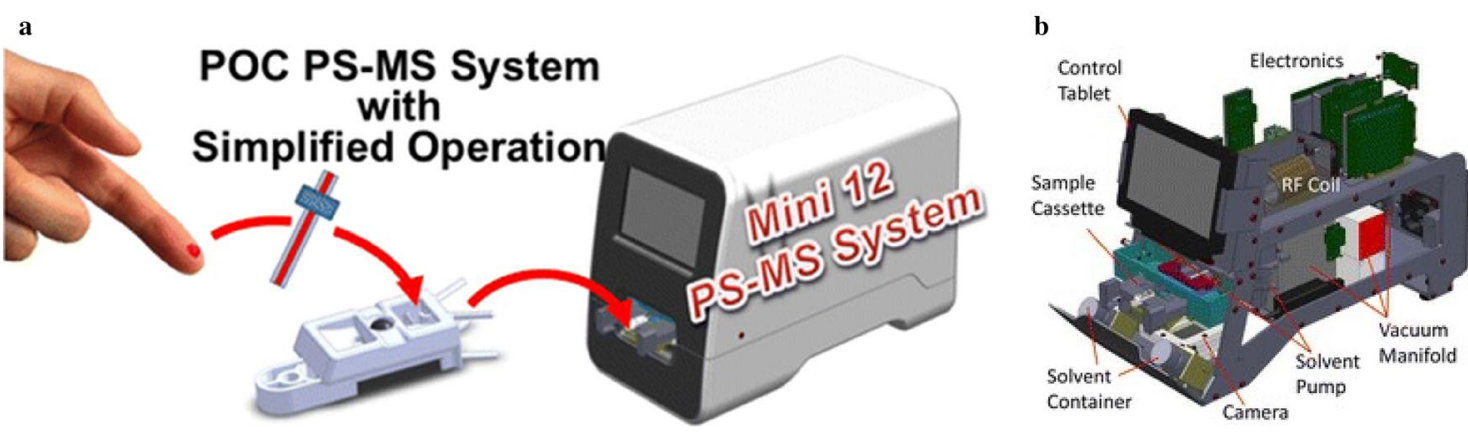

Fig. 12 Mini 12 paper-spray (PS) MS developed by Li et al. [118] a Simplified user operation for point-of-care (POC) applications. b Overview of system components 
be considered as a more sensitive detection method. It is important to note that this debate links back to an earlier point; one must establish the intended purpose and the minimum requirements for satisfactory results. Besides improving separation efficiency, a parallel objective should be to validate minimal separation efficiencies that can maintain accurate results [128].

\section{Portable LC Platforms 1980-2020}

The pursuit of portable systems has a long and varied history, both capitalising on advances in the wider field while also driving its own. A timeline of the portable LC instruments to date is shown in Fig. 13 differentiated by their weight, an integral measure of their portability. Here on in, we will explore these systems, evaluating their capabilities and highlighting their unique contributions to the field. In addition, a radar chart is presented for each assessing the system according to key aspects of the BETTER criteria.

\section{Otagawa et al. (1986)}

Otagawa et al. were among the first to report the use of a portable HPLC [134]. However, this is not straightforward to confirm since details of the system are sparse and the overall dimensions, weight and power requirements of the system were not reported. An isocratic system was used for the analysis of primary aromatic amines (PAA) in coal-derived materials onsite. The system comprised a direct current (or $6 \mathrm{~V}$ battery) powered dual-piston isocratic pump capable of flow rates between 0.22 and $0.58 \mathrm{~mL} / \mathrm{min}$; a pulse dampener with built-in pressure gauge; an automatic continuous-flow sample injector $(20 \mu \mathrm{L})$; a reverse-phase (RP) column $(10 \mu \mathrm{m}, 3 \mathrm{~cm} \times 4.6 \mathrm{~mm}$ I.D. $)$; and a thin-layer transducer cell containing a glassy carbon working electrode as part of the ECD system outputting to a strip-chart recorder (Fig. 14a).

The system's analytical capability was assessed analysing a 600 ppm raw coal oil sample. The system identified 18 peaks with no baseline separation and poor resolution, limiting the ability to quantify components (Fig. 14b). Otagawa et al. recognised this in comparing data GC or GC-MS system in which the identification of over 130 components was possible. The reported detection limits for the PAA's were in the 2.4-3.3 ppb range, indicating respectable sensitivity and specificity. However, the ECD was highly sensitive to changes in flow rate, temperature, $\mathrm{pH}$ and electrode contamination. Sensitivity to changes in these factors indicate low data reproducibility and suggest that the detector is unsuitable for use in a portable device designed for field testing [56]. In addition, the analytical capability of the instrument is limited by the low attainable pressure $(<10$ bar $)$ and consequently restricts the choice of chromatographic columns. Low pressure pumping systems constrain the flow rates resulting in extended analysis times. Therefore, narrow bore high efficiency columns cannot be used since the flow rates achieved by the pump are too low.

This early iteration of portable HPLC showcased the likely potential and apparent limitations which would later be overcome. The applicability of the system for field or point-of-sampling analysis is restricted by the use of a conventional RP column found in commercial laboratory instruments, as well as the use of an ECD system. Incorporating a $4.6 \mathrm{~mm}$ I.D. conventional column reduces the

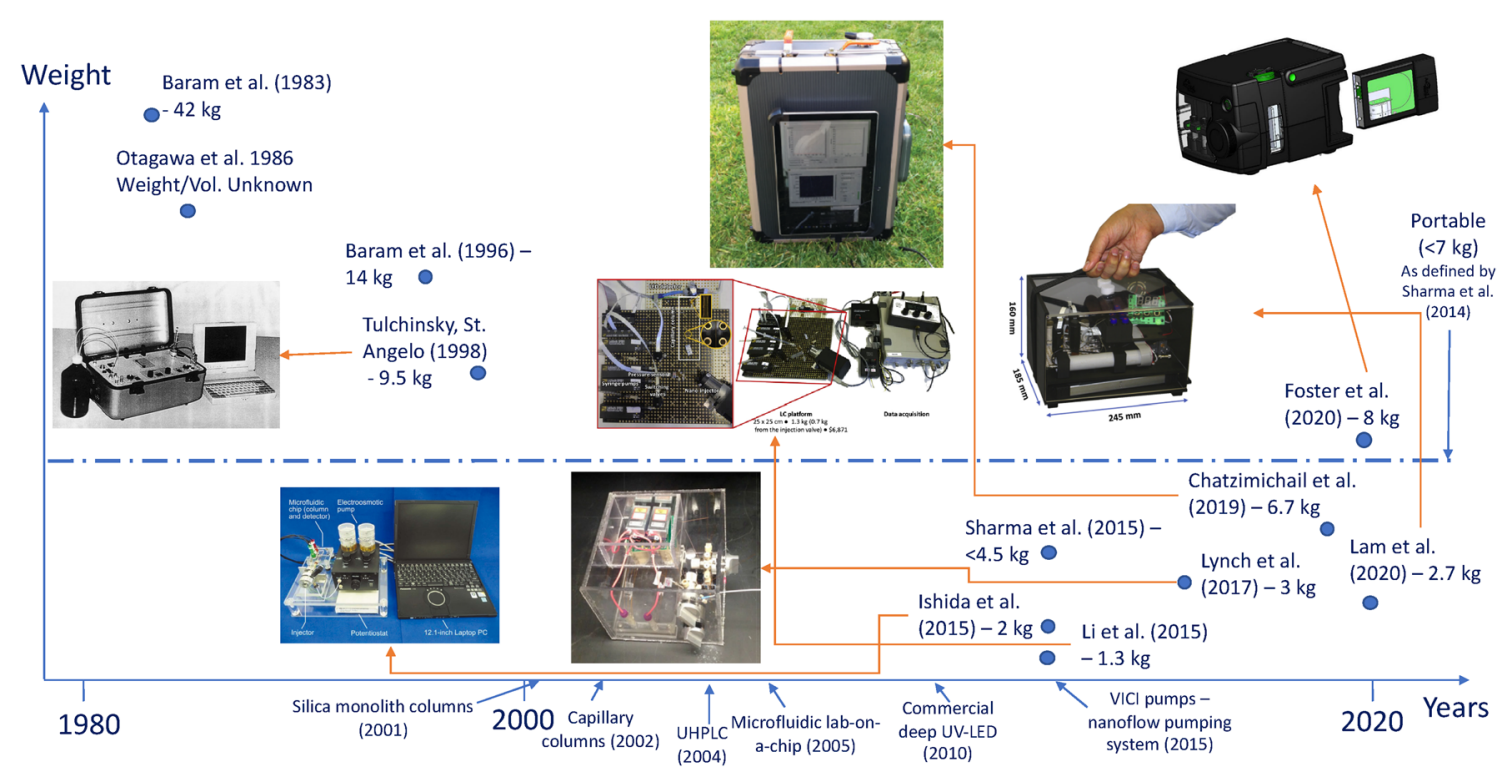

Fig. 13 Timeline of portable HPLC devices from 1980 to present accounting for emergence of new technologies [58, 129-133] 
a

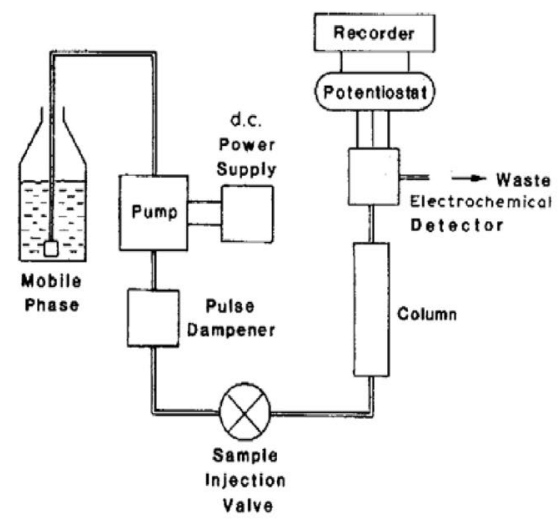

b

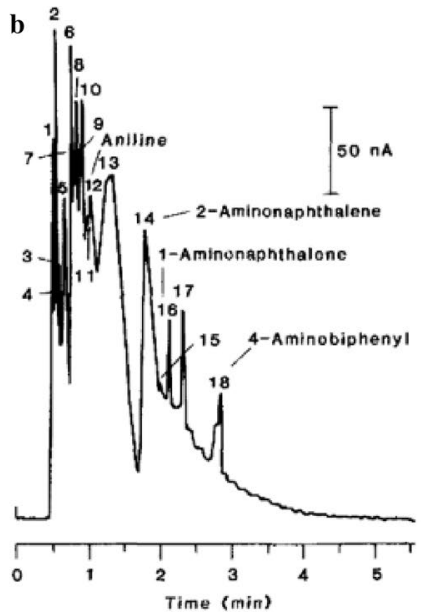

c

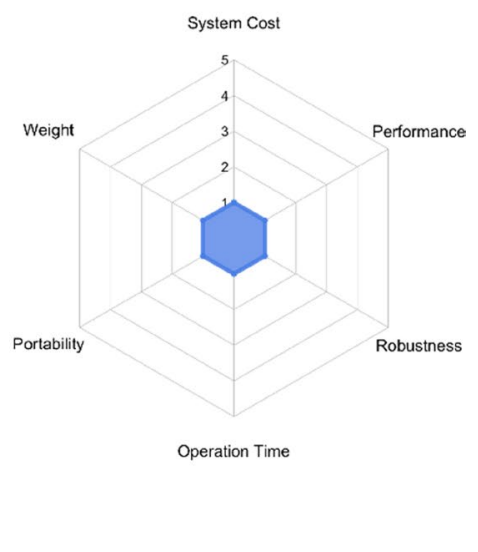

Fig. 14 Portable HPLC system by Otagawa et al. [134] a Schematic b Chromatogram of $600 \mathrm{ppm}$ raw coal oil sample at a flow rate of $0.22 \mathrm{~mL} /$ min. $\mathbf{c}$ Radar chart assessing the system according to the BETTER criteria

portability of the device due to increased solvent consumption and waste generation, subsequently increasing the size and weight of the system. The majority of HPLC detectors are concentration dependent. Therefore, the detector signal, and consequently the maximum peak height for an analyte, is directly proportional to the concentration of the analyte in the detector cell [135]. This means that by using a $4.6 \mathrm{~mm}$ I.D. column as compared to a micro or capillary column, the analyte dilution is greater and therefore, the resulting peaks are smaller. This is because the dilution factor is directly proportional to the volume of the column, overall resulting in lower sensitivities. This could consequently pose problems when analysing samples where analytes are present at trace concentrations.

No comments were made on cost or the robustness of the systems. The device made use of an ECD-this detection system has high power requirements and most likely needed a heavy battery pack to power it for any reasonable length of time. Conventional wide bore columns (4.6 mm I.D.) were used with comparatively greater solvent consumption necessitating large solvent reservoirs.

\section{Baram et al. (1996)}

The first LC system sold commercially as a portable solution was the OB-4 or MiLiChrom (Micro Liquid Chromatograph), reported by Baram et al. in 1983; this device is better referred to as a lab-station portable system since the system weighed approximately $42 \mathrm{~kg}$ and required a regular $\mathrm{AC}$ power supply. The system had dimensions of $70 \mathrm{~cm} \times 30 \mathrm{~cm} \times 50 \mathrm{~cm}(\mathrm{~L} \times \mathrm{W} \times \mathrm{H})$ and employed a multi-wavelength photometric detector. The portability of the OB-4 may be questioned with respect to today's definition, however, it is important to note that the OB-4 was commercially sold until 1996 with over 5000 devices produced $[55,136]$. In the decade following its conception, Baram et al. reported a follow-up field chromatograph, the MiLiChrom-4, weighing $14 \mathrm{~kg}$ with dimensions $53 \mathrm{~cm} \times 20 \mathrm{~cm} \times 30 \mathrm{~cm}$ (Fig. 15a) [55]. The weight and size achieved by the device is commendable given the limited availability of miniaturised components at the time of conception. Currently, for an LC system to qualify as portable, it must weigh $<7 \mathrm{~kg}$. [56] Since this system nominally weighs twice what is expected from a portable system and is not battery-operable, it is difficult for the MiLiChrom-4 to be considered a portable LC. Nevertheless, the MiLiChrom-4 was a significant step forward for portable LC at the time and, although now clearly dated, its performance would still stand up reasonably well to its contemporaries.

The gradient enabled system comprised two syringe pumps capable of flow rates between 5 and $1000 \mu \mathrm{L} / \mathrm{min}$; an automatic stop-flow injector; RP column $(6-8 \mathrm{~cm} \times 2 \mathrm{~mm}$ I.D.). The detector was a deuterium UV-lamp based microspectrophotometer (Fig. 15b). Single wavelength detection hampers modern portable instruments that use LEDs, whereas the instruments reported by Baram et al. could scan 190-360 nm. Of course, their setup required a number of optical elements that would make portable operation challenging. Similar to its predecessor, the MiLiChrom-4 was limited in its requirement for mains power, restricting its use to mobile laboratories with such capability.

Baram used the device to demonstrate the separations of various environmental contaminants including pesticides, polynuclear aromatic hydrocarbons, phenols, and phthalate esters as well as rapid separations $(<4 \mathrm{~min})$ of polynitro explosives (Fig. 16) [55]. Chromatographic peaks appear well-resolved with good baseline separation and high SNR. 


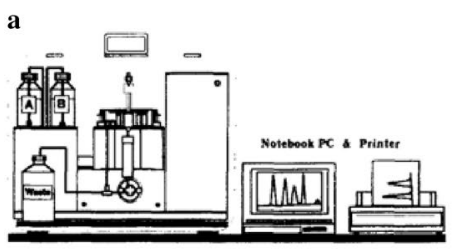

b

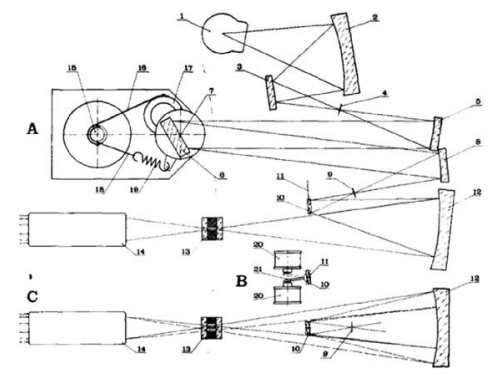

c

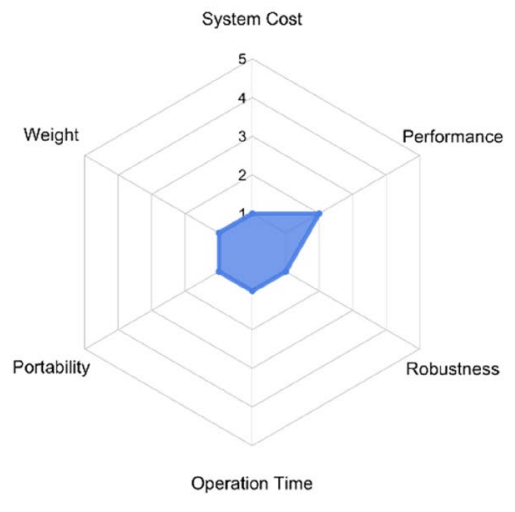

Fig. 15 a Schematic of portable HPLC system by Baram et al. [55] b Optical setup of the detection system in the OB-4 chromatograph with (A) top view, (B) schematic of the electromagnetic drive of the
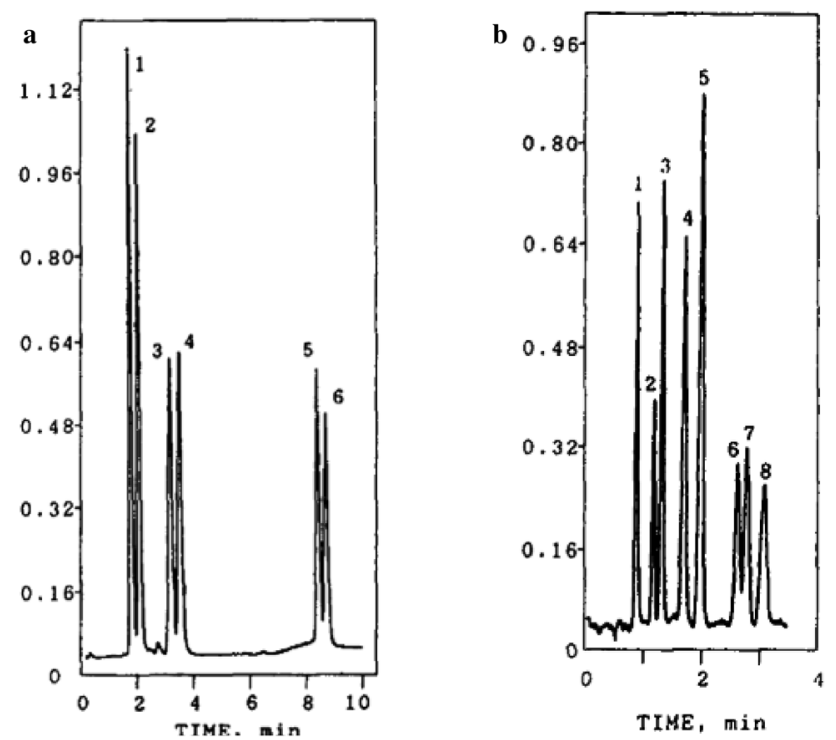

Fig. 16 a Gradient separation of phthalate esters b isocratic separation of polynitro explosives. Peak identification: a (1) dimethyl phthalate; (2) diethyl phthalate; (3) di-n-butyl phthalate; (4) butyl benzyl phtha-late; (5) bis(2-ethylhexyl) phthalate; (6) di- $n$-octyl phthalate. b (1) HMX; (2) RDX; (3) TNB; (4) tetryl; (5) TNT; (6) 2,4-DNT; (7) 2,6-DNT; (8) picric acid. Reproduced from Baram et al. [55]

Baram set out a number of criteria (as per the BETTER criteria above) considered to be requirements for LC instruments in the field, including resistance to vibration [55]. However, no comment or tests were made in support of the robustness of the MiLiChrom-4. From the components and their construction, it is unlikely to have been resistant to mechanical shocks typical of field use and transportation. mirror, and (C) side view. $\mathbf{c}$ Radar chart assessing the system according to the BETTER criteria Reproduced from Baram et al. [136]

\section{Tulchinsky, St. Angelo (1998)}

Towards the end of the twentieth Century, another portable HPLC device known as "Minichrom" was reported by Tulchinsky and St Angelo [137, 138]. It marked a step change from its predecessors in that it attempted to unify components into a single casing (Fig. 17a). The system is enclosed in an aluminium case reported to be capable of withstanding shocks and vibrations common in field testing. It is crucial for measures to be put in place to protect the sensitive components of the system. Since there were no published results of mechanical stability or impact analysis for Minichrom, the robustness of the system was not confirmed.

The Minichrom incorporated a fixed-wavelength UV detector using various light sources including $\mathrm{Zn}, \mathrm{Cd}$ and W lamps; two dual-piston reciprocating pumps with damper capable of isocratic and gradient elution and a maximum pressure of 350 bar (5000 PSI); and a conventional RP column (4.6 mm I.D.). The flow rates achieved by each pump were between 0.1 and $2.5 \mathrm{~mL} / \mathrm{min}$. The system shown in Fig. 17a does not include the power source, a lead-acid battery; typically, these weigh $15 \mathrm{~kg}$ alone.

The system was used to demonstrate the separation of phenolic pollutants in lake water near industrial areas of Leipzig, Germany. Both chromatograms present limited analytical performance resulting from low resolution and a lack of baseline separation of the peaks (Fig. 17b).

The Minichrom system presented an important advance in portable HPLC with the 350 bar maximum pressure achieved. Analytical drawbacks of the system exist, nonetheless. The dual-piston reciprocating pumps, while robust and able to produce gradients, produce pulsations at low flow rates. Although this effect is marginally reduced by programming the pumps $180^{\circ}$ out of phase from one another, pulsations persist. The pump arrangement adopted in Minichrom 
$\mathbf{a}$

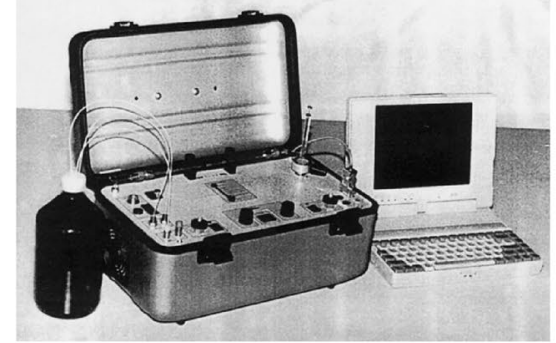

b

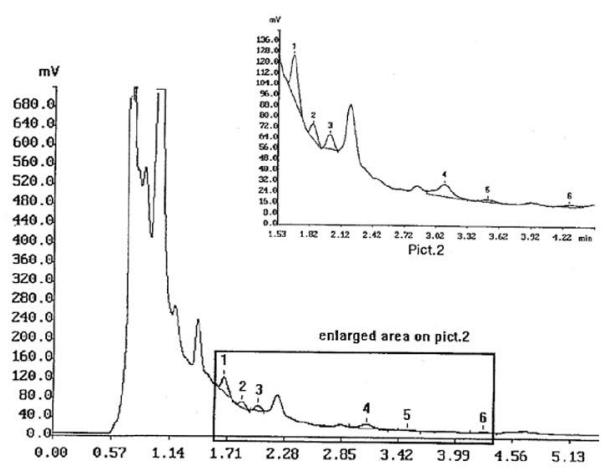

c

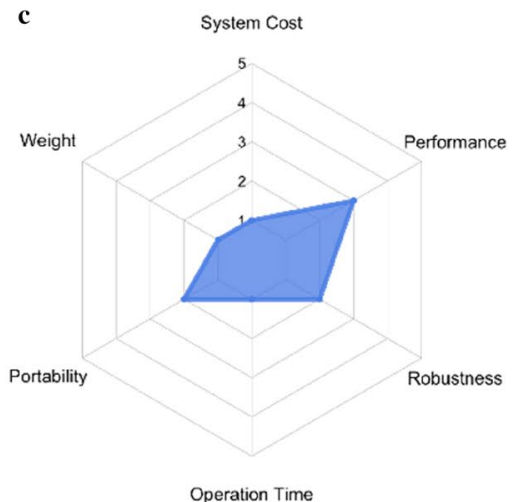

Fig. 17 a Standard Minichrom setup. b Isocratic separation of phenolic pollutants in lake water. $\mathbf{c}$ Radar chart assessing the system according to the BETTER criteria. Reproduced from Tulchinsky et al. [138]

allows a lower flow rate range for the pump [139]. Low LODs were achieved for phenol $(0.2 \mathrm{ppm})$ and anthracene $(0.015 \mathrm{ppm})$ demonstrating the sensitivity of the system.

This device is smaller and reportedly lighter $(9.5 \mathrm{~kg})$ than previous generations introduced by Baram or Otagawa, mainly owing to use of a more compact fixed-wavelength detector. It is worth mentioning that the reported size and weight of Minichrom does not account for the solvent bottles, computer and battery necessary for its operation.

Tulchinsky et al. discussed the cost of the system briefly. Utilising a fixed-wavelength UV detector reduces the overall cost of the device, allowing the demands of modern fieldmonitoring to be met with economic cost of the system in mind. However, this brief discussion does not provide a conclusive idea of the likely capital or running cost of the system. Therefore, it is difficult to discern whether Minichrom satisfies the cost criterion of a portable system. The purpose of Minichrom was to be commercialised, however, no evidence of large-scale commercial fabrication can be found. A possible theory may be that the decreasing size and weight of commercial laboratory LC systems as well as substantial advances in the form of UHPLC in the decade following the conception of Minichrom meant that this device appeared less attractive.

Perhaps these are overly critical assessments if we are to consider the times in which the systems discussed thus far were developed. They precluded the advent of technologies such as capillary columns, microfluidics, miniaturised high-pressure pumping systems, and deep UV-LED detectors, to name a few. The introduction of these components transformed the development of portable HPLC equipment, as will be evident as we proceed through this review.

\section{Ishida et al. (2015)}

Ishida et al. reported a compact system comprising an EOP; microfluidic-chip based RP column $(3 \mathrm{~cm} \times 0.8 \mathrm{~mm}$ I.D. $)$ packed with C18 silica particles; manual injector; USBpowered potentiostat; and an ECD [22]. Notably, this device was the first of its kind with a fully integrated system featuring a microfluidic chip (Fig. 18). The size $(18 \mathrm{~cm} \times 26$ a

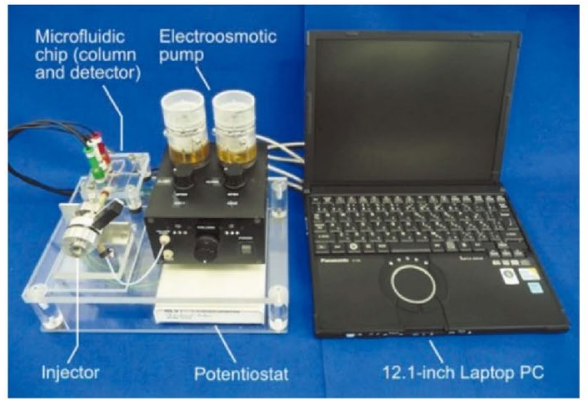

b

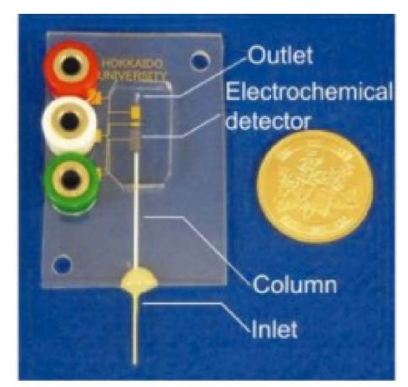

c

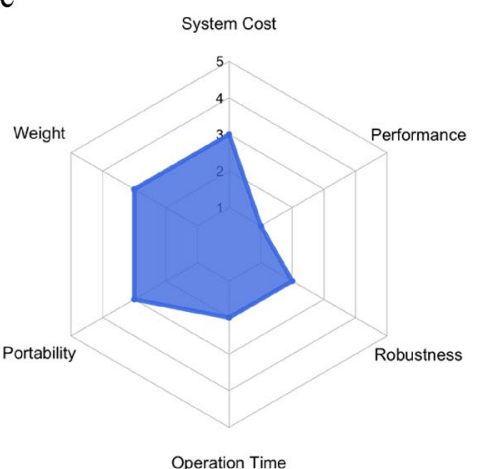

Fig. 18 a Portable HPLC system b microfluidic chip device by Ishida et al. [22] c Radar chart assessing the system according to the BETTER criteria 
$\mathrm{cm} \times 21 \mathrm{~cm}-\mathrm{L} \times \mathrm{W} \times \mathrm{H})$ and weight $(2 \mathrm{~kg})$ of the fluidic system were significantly smaller/lighter owing to the use of a microfabricated EOP, column and ECD. The microfluidic LC, not including the requisite computer system (approx. an additional 2-3 kg inferred from Fig. 18a), remains one of the lightest instruments to date.

The detection method used by Ishida has previously been employed in a portable LC [134]. One of the main issues in the previous device was related to the size of commercial ECDs, and the impact on the overall dimensions and weight of the device. By fabricating the ECD onto the microfluidic chip, a substantial reduction in size could be accomplished. As a result, ECDs are becoming commonly used in lab-on-a-chip technology [56, 140-142].

The advent of microfluidics has enabled substantial miniaturisation of instrumentation as well as integration of key components onto a single chip. These systems offer low dead-volume connections, ease of automation, and are appropriate for low sample volumes [143]. The battery-operated system was used in the isocratic separation of alkylphenols, catechin, catecholamine, and amino acids. However, the system was restricted by the inability to achieve LC pressures $(<10$ bar) resulting in low flow rates $(5 \mu \mathrm{L} / \mathrm{min})$ and long separation times (15-40 min). While gradient-elution can be achieved by the EOP, only isocratic separation was demonstrated. The chromatograms of the alkylphenol and catecholamine separations presented show signs of band broadening and limited baseline separation (Fig. 19).

EOPs certainly have the potential to support miniaturised high-pressure pumps. The pumping system designed by Ishida has low power consumption in comparison to other

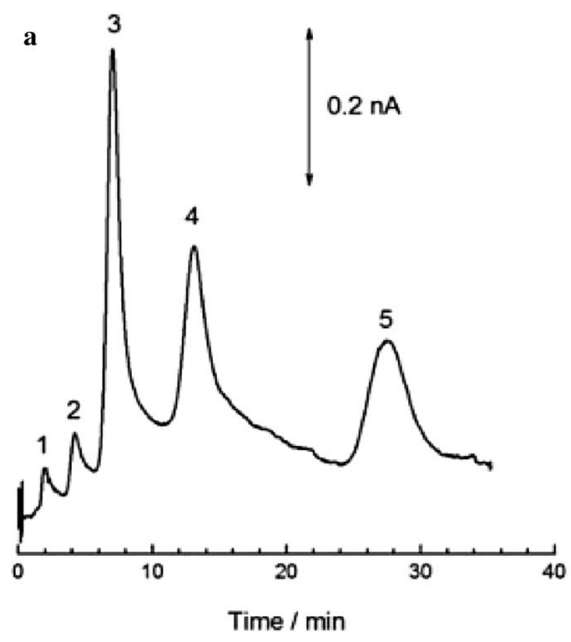

Fig. 19 Gradient mode separations of a alkylphenols, and $\mathbf{b}$ catecholamine using the portable HPLC system by Ishida et al. [22] Peak identification: a (1) phenol; (2) 2-methylphenol; (3) 2-ethylphenol; (4) 2-propylphenol. b (1) noradrenaline; (2) adrenaline; (3) dopamine. Analytical conditions; a mobile phase: A: $0.1 \mathrm{M} \mathrm{NaClO} 4, \mathrm{~B}$ :
EOPs but cannot achieve pressures necessary for HPLC. The pump presented here could not exceed 10 bar. While EOPs have been reported which can generate pressures exceeding 1200 bar, these require several kilovolts to power necessitating bulky external power supplies, currently difficult to achieve in a portable system [66, 67]. However, Ishida et al. believe that their EOP system can be operated on battery for up to $24 \mathrm{~h}$ at a flow rate of $5 \mu \mathrm{L} / \mathrm{min}$.

The cost of the system was not discussed, however, the system benefits from the use of a microfluidic chip-based LC. Microfluidic methods traditionally employ cost-effective, and compact chips intended for fast and precise analysis of small sample volumes [144]. Similarly, the robustness and ease-of-use of the device were not discussed; however, some sensible conclusions can be drawn based on the components used. As mentioned previously, the monolithic advantage that integrated microfluidic bring can also be a major flaw if a breakdown of a single component occurs; often compelling the replacement of the entire device.

The stability of the portable LC within a field environment was not part of the tests conducted with this device. An issue that may be encountered is related to the stability of the EOP in response to shocks and vibrations. Flow rate fluctuations remains the main limitation to the commercialisation of EOP-based portable devices. Variations can arise from unstable voltage sources, and chemical breakdown within the pumping element [139]. Despite this, since EOPs do not contain moving parts, one could expect a degree of resilience to mechanical damage.

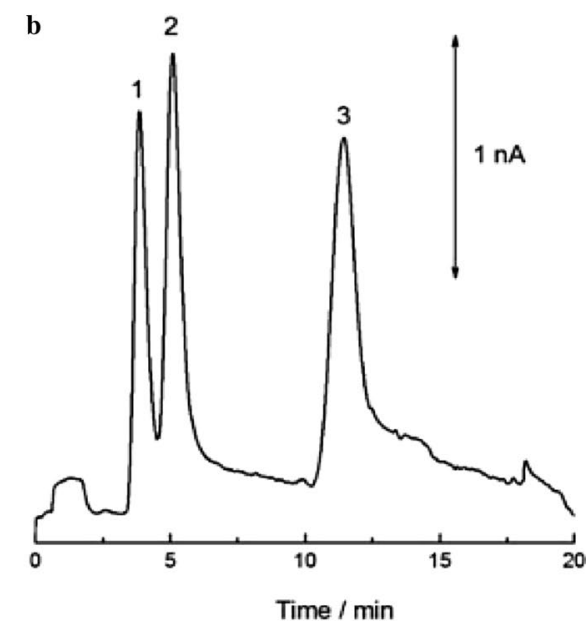

methanol; $\mathrm{A}: \mathrm{B}=1: 1(\mathrm{v} / \mathrm{v})$; flow rate: $5 \mu \mathrm{L} / \mathrm{min}$; detection: $+0.6 \mathrm{~V}$ vs. Au. b mobile phase: A: $50 \mathrm{mM}$ phosphate, $50 \mathrm{mM}$ citrate, $100 \mathrm{mg} / \mathrm{L}$ octanesulphonate, $40 \mathrm{mg} / \mathrm{L}$ EDTA-2Na (pH 3.0) B: methanol; $\mathrm{A}: \mathrm{B}=95: 5(\mathrm{v} / \mathrm{v})$; flow rate: $5 \mu \mathrm{L} / \mathrm{min}$; detection: $+0.6 \mathrm{~V}$ vs. Au 


\section{Sharma et al. (2015)}

Two prototype portable LC devices were produced by Sharma et al. in 2014 and 2015. The first system reported an isocratic piston-based pumping system integrated with a stop-flow injector and an on-column $\mathrm{Hg}$ lamp (fixed-wavelength) UV-absorption detector. Separation of six benzene compounds (uracil, toluene, ethylbenzene, propylbenzene, butylbenzene and amylbenzene) using an isocratic method was demonstrated within an analysis time of $19 \mathrm{~min}$. The system displayed good separation ability with low LODs achieved using the on-column detector (40 ppb), and the pumping system produced low flow rates in the $\mathrm{nL} / \mathrm{min}$ flow range in combination with capillary columns. The lampbased detector limited the potential of the instrument and so in a follow-up paper, Sharma et al. miniaturised the detector by employing a deep UV-LED $(260 \mathrm{~nm})$ and led to a reduction in LODs by up to two orders of magnitude. With the advantages of low power requirements, stable light output and portability, LED-based detectors have earned significant attention, especially in portable LC instrumentation.

The second, and most recent, system reported a gradient nanoflow pumping system combined with the on-column, fixed-wavelength, and UV-LED detector (Fig. 20a). The main modification from the previous prototype involved modifying the pumping system from a single to a dual pump system enabling programmable gradient elution. The system comprised two commercial nanoflow pumps constructed by VICI Valco Instruments (Houston, TX, USA) capable of up to 550 bar pumping pressure controlled by micro stepper motors; a three-way static mixing tee connected to a serpentine tube $(1.3 \mathrm{~cm} \times 0.025 \mathrm{~cm} \mathrm{I.D.)}$ for solvent mixing; a high-pressure valve; a RP monolithic PEGDA column
(13 $\mathrm{cm} \times 150 \mu \mathrm{m}$ I.D.); and a stop-flow injector $(60 \mathrm{~nL})$. The high pressures that can be reached are impressive given the size of the system, $31 \times 18 \times 14 \mathrm{~cm}(\mathrm{~L} \times \mathrm{W} \times \mathrm{H})$. It is important to mention that this is the first portable LC to use a capillary column. Despite the introduction of capillary columns in the early twenty-first century [130], previous LC systems due to their pumping limitations have relied on wide-bore columns.

This system fabricated by Sharma et al. successfully fulfils the majority of the essential requirements set out previously, with the exception of integrating all components into a single unit, and the durability of the device since no testing was conducted into the stability following environmental fluctuations. However, it must be noted that the system was a prototype designed to demonstrate the performance of the pumping system. Its chromatographic performance was characterised using a three-component pesticide mixture as well as a mixture of five phenols. The chromatogram shows good peak shapes with baseline separation achieved in under 20 min (Fig. 20b).

The separation of complex mixtures of compounds with a wide variety of polarities is difficult to achieve with isocratic methods. Therefore, more powerful gradient modes are used to expand the capabilities and applications of portable LC systems. The performance of a gradient LC system is defined by the gradient accuracy and precision which establishes the separation reproducibility. Within day-to-day experiments, the gradient step accuracy $(<0.74 \%$ deviation from predicted values); gradient step precision $(\mathrm{RSD}<1.2 \%, n=4)$; and comparison of the retention times of a pesticide mixture revealed high reproducibility ( $\mathrm{RSD}<1.2 \%, n=4$ ). Previous systems have reported gradient capability, however, these
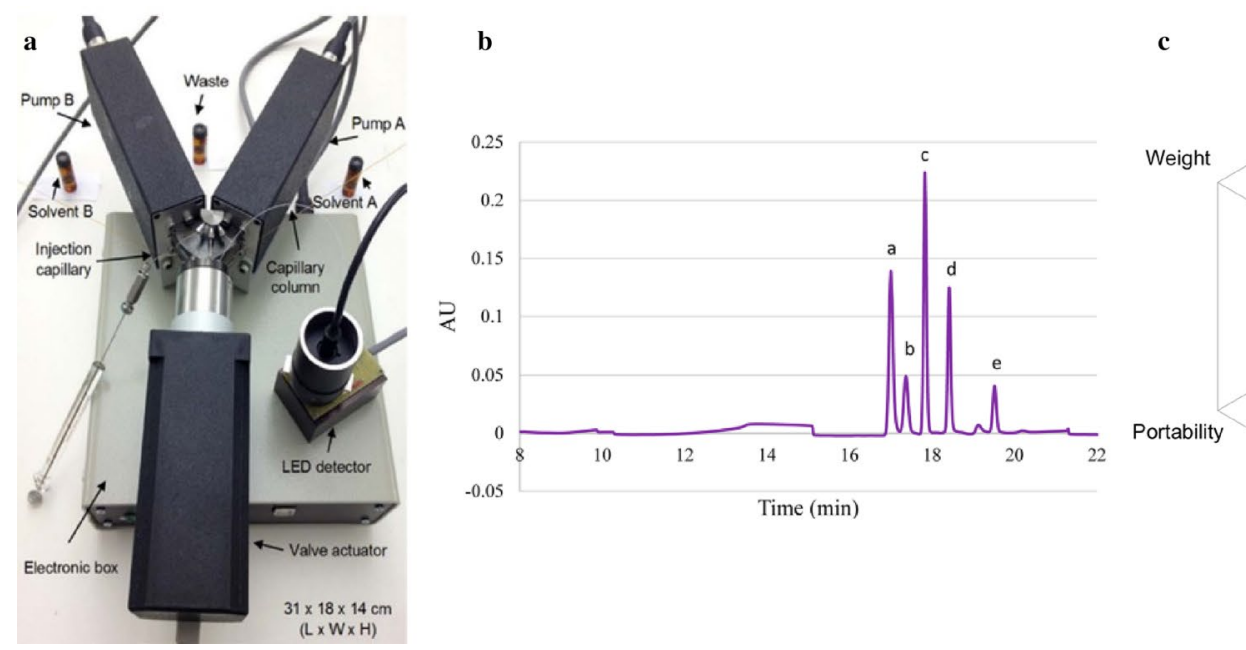

Time $(\min )$

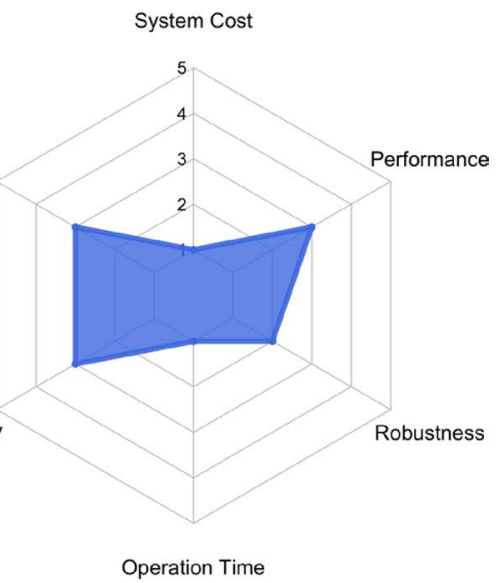

Fig. 20 a Hand-portable HPLC system developed by Sharma et al. [58] utilising a miniaturised LED detector. b Gradient separation of a mixture of phenols. Peak assignments are $a$ : phenol, $b$ : 2-nitrophe- nol, $c$ : 4-nitrophenol, $d$ : 2,4-dichlorophenol, and $e$ : 2,4-dinitrophenol. c Radar chart assessing the system according to the BETTER criteria. Reproduced from Sharma et al. [58] 
systems reported limited information on gradient performance $[22,138]$.

The LC system weighed around $4.5 \mathrm{~kg}$, which is considerably less than the requirements outlined previously for a portable device. However, batteries and a temperature control device are missing from the system; these will undoubtedly increase the size and weight. The system was battery-operable using a $10 \mathrm{~A}-\mathrm{h} 24 \mathrm{~V}$ DC battery, weighing around $2-3 \mathrm{~kg}$, capable of powering the device for up to $8 \mathrm{~h}$. This battery type also finds use within electrically powered bicycles. The demanding power requirements are likely a result of the custom pumps used for precise gradient control. While the system can be battery-powered, a regular $120 \mathrm{~V}$ $\mathrm{AC}$ power line and voltage converter was used during the tests reported.

The cost of the device was not part of the instrumentation discussion; therefore, while no definitive conclusions can be formed, some speculative statements will be debated. The pumping system employed is a custom design from VICI Valco Instruments; this is likely to be expensive and where the bulk of the prototype cost lies. Although the pumps provide excellent performance in a compact design, the cost of the commercialised portable device will likely exceed that of modern laboratory-based instruments as a result. Of course, as discussed previously, instruments with high capital costs will find it hard to find widespread adoption.

\section{Li et al. (2015)}

In the same year, Li et al. endeavoured to develop a portable LC system, capable of gradient elution, by exploring a different strategy. The majority of portable LC designs discussed so far, while progressive and unique, have generally been constructed in-house using parts and components with limited availability to the end-user. An open-platform modular design taking advantage of off-the-shelf microfluidic components provides the opportunity to alter and improve specific features, without sacrificing portability and cost. In recent years, considerable progress has been made in the field of miniaturisation of pumps applicable to liquid chromatography resulting in several commercial entities emerging including LabSmith Inc. (Livermore, CA, USA) [145], The Lee Company (Westbrook, CT, USA) [146], and Sciex (Framingham, MA, USA) [147]. The arrival of these miniature pumps has enhanced the potential of portable HPLC instrumentation closer to the concept of $\mu$ TAS.

The Li system was constructed using off-the-shelf LabSmith pumps; a VICI valco nano-injector $(20 \mathrm{~nL})$; an oncolumn LED-based photometric detector; and a Chromolith CapRod RP-18 monolith column (180 mm × $100 \mu \mathrm{m}$ I.D.). The non-splitting pumping system comprised four programmable microsyringe pumps ( $5 \mu \mathrm{L}$ volume each); two fourport switching valves; and a pressure sensor assembled on a breadboard. Two sets of micro-syringe pumps were each connected to an automated microfluidic four port switching valve such that one pump injects the mobile phase while the other replenishes. This overcomes the limited volumes syringes can dispense, enabling a near uninterrupted flow of eluent to be delivered more akin to a conventional dualpiston pump. Each mobile phase is dispensed by a pair of these pumps, resulting in four pumps with fixed volume capacities coupled using an interconnect cross to combine microfluidic flows (Fig. 21a). The system was reported to achieve high pressures. However, these syringe pumps are not designed for precision flow or high-pressure applications a

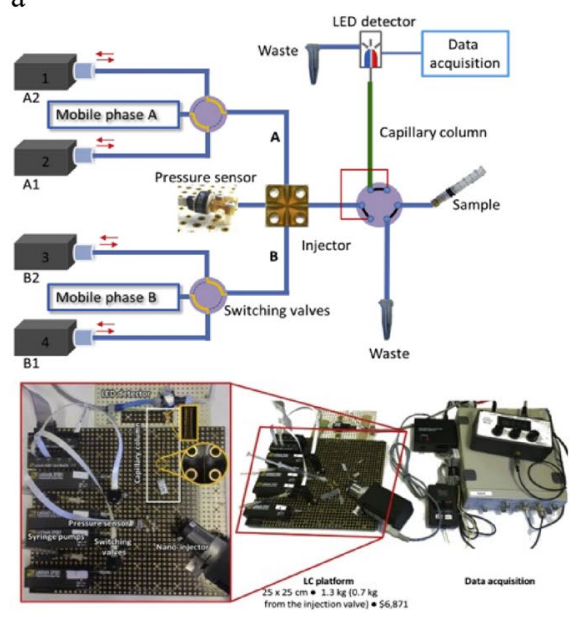

$\mathrm{b}$

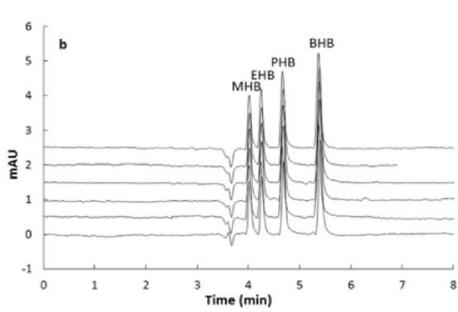

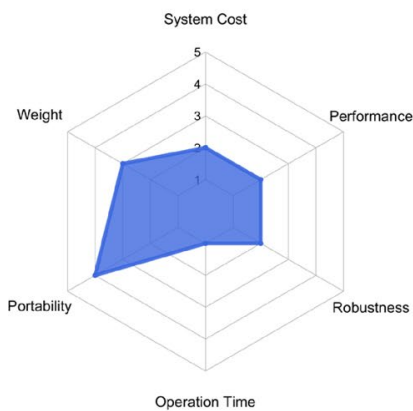

c
Fig. 21 a Schematic and photograph of the portable medium-pressure LC system. b Isocratic separations of a parabens mixture: 4-hydroxybenzoate (MHP), ethyl 4-hydroxybenzoate (EHB), propyl 4-hydroxy- benzoate (PHB), and butyl 4-hydroxybenzoate (BHB). c Radar chart assessing the system according to the BETTER criteria Reproduced from Li et al. [65] 
since there is considerable variation between pumps in manufacture and all suffer from pronounced oscillatory flow profiles. These issues are not covered in published accounts. In our laboratory, they find use in low pressure $(<10$ bar $)$ microfluidic applications; when tested with a commercial monolith column $(150 \mathrm{~mm} \times 100 \mu \mathrm{m}$ I.D.; Merck Chromolith) the syringes ( $20 \mu \mathrm{L}$ and $80 \mu \mathrm{L}$ volume) had a high tendency to stall, making this technology unsuitable for our field applications.

The analytical capability of the system was assessed by characterising the maximum operating pressure of the miniature pumps, and their performance in isocratic and gradient separations. The actual flow rates were determined experimentally in relation to the set flow rate. Nevertheless, the system is compatible with short, low back pressure columns. The systems performance to isocratically separate food dyes and parabens, as well as using a gradient for the dyes, was assessed (Fig. 21b). Four parabens were separated with baseline resolution within 6 min. Over six consecutive runs, the RSDs of retention times were between 0.1 and $0.2 \%$, and the peak areas between 0.5 and $1.0 \%$. Gradient mode separations were performed with either methanol or acetonitrile mobile phases. Satisfactory resolution and SNR were achieved in $8 \mathrm{~min}$ with a mixture of up to seven dyes as well as a void volume marker. The complete selection of dyes was identified with the acetonitrile mobile phase. Comparison with the isocratic separation highlights narrower peaks and higher peak capacity using gradient mode. Over eight consecutive runs, the RSDs of retention times were between 1.2 and $4.6 \%$, and the peak areas between 3.2 and $4.6 \%$.

This system was laudable for its use of open hardware and discussion of the overall cost of the system. The cost of the system is an essential factor that must be considered; $\mathrm{Li}$ et al. specified a cost of $\$ 6871$ for the modular LC platform. This is considerably less expensive than a commercial laboratory HPLC system which can cost in a region exceeding
$\$ 50,000$. The Labsmith LC platform (Fig. 21a) has dimensions $25 \mathrm{~cm} \times 25 \mathrm{~cm}(\mathrm{~L} \times \mathrm{W})$ and a weight of $1.3 \mathrm{~kg}$. It is important to note that this does not include any power or data acquisition modules, although this need not be much given the ubiquity of single board microcontrollers and lithium-ion batteries. The system was an open bench prototype and so was not encased in any packaging. Since the pumping system, column, nano-injector, and on-column detector have a relatively small footprint, fabricating an appropriate housing to withstand damage from shocks and vibrations should not be too difficult.

\section{Lynch et al. (2017)}

Previously, Ishida et al. integrated an electroosmotic pumping mechanism into a portable chip-based LC [22], however, the system was restricted by low attainable pressures. Advances in EOPs that are capable of attaining high pressures are being capitalised in the development of miniaturised portable LC [67, 148-151]. In 2017, Lynch et al. reported the HPLC cartridge which incorporated a series of stacked EOPs alongside a network of valves to drive the separation of complex sample mixtures, all within a small footprint measuring $20 \mathrm{~cm} \times 20 \mathrm{~cm} \times 17.5 \mathrm{~cm}(\mathrm{~L} \times \mathrm{W} \times \mathrm{H})$ and weighed approximately $3 \mathrm{~kg}$.

The system (Fig. 22a) comprised two EOP units powered by a custom in-house designed high-voltage power supply (HVPS); a 12-port switching valve, a ten-port stream selector, a $60 \mathrm{~nL}$ injection valve (all obtained from VICI Valco Instruments); and a Waters Atlantis dC18 NanoEase monolithic capillary column $(100 \mathrm{~mm} \times 75 \mathrm{~mm}$ I.D.). Each EOP unit serves a specific purpose: one infuses mobile phase to occupy the eluent loops, while the other drives the mobile phase towards sample separation. Each EOP unit was able to generate a pressure close to $140 \mathrm{bar}$ at $5 \mathrm{kV}$. The pumping pressures and flow rates could be altered by adjusting the voltages applied. The ten-port stream selector connects

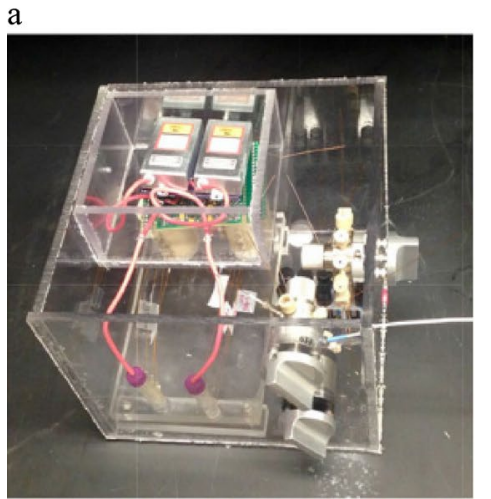

$\mathrm{b}$

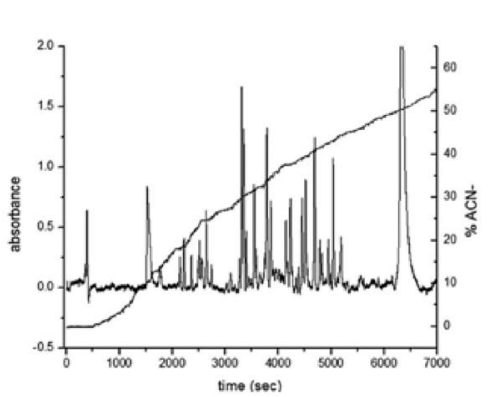

C

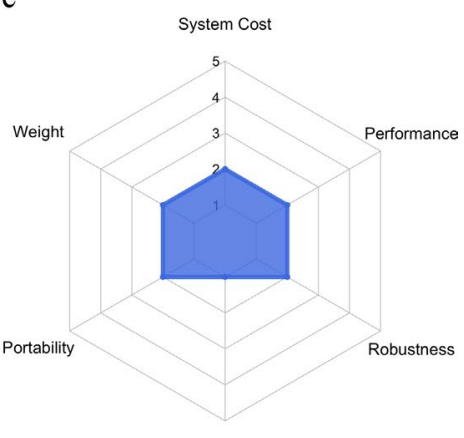

Operation Time

Fig. 22 HPLC cartridge fabricated by Lynch et al. [59] a Photograph of cartridge. b Gradient separation of a myoglobin digest at a flow rate of $150 \mathrm{~nL} / \mathrm{min}$ based on a 20-nL sample injection volume. $\mathbf{c}$ Radar chart assessing the system according to the BETTER criteria 
to nine eluent solutions allowing several gradient profiles to be generated by varying the eluent compositions. A detector was not integrated into the system, and as such cannot be considered a fully integrated portable HPLC device.

The authors state that the versatility of the cartridge is brought about by its ability to couple to a variety of detection systems. The desired outcome of this work is to produce a HPLC cartridge with interchangeable detectors based upon the analyte of interest. This modular design, perhaps inspired by Li et al. [65], may be a good approach to widening adoption since different detection systems are suited to different analyses. Lynch et al. demonstrated the chromatographic capability of their system separating peptide and protein mixes and coupling to a UV absorbance detector and a mass spectrometer, the two most commonly used detection strategies.

The tryptic digests of BSA and myoglobin were separated using a gradient profile within $120 \mathrm{~min}$, with separation profiles comparable to those obtained on a commercial Agilent HPLC (Fig. 22b). Experiments were conducted to monitor the gradient profile and its reproducibility in conjunction with a contactless conductivity detector. Repeat runs $(n=3)$ appear to have generated a similar profile, in the absence of RSD values. The reason for this may lie in flow rate fluctuations as a result of electrode or monolithic column degradations over numerous runs. Without gradient reproducibility statistics including baseline repeatability and RSDs of retention times and peak areas, an assessment of the performance is difficult to come by. This complicates comparison with other portable HPLC systems. In addition, the separation performance of the cartridge is unclear since the number of expected elements were not specified. Therefore, although several narrow, baseline separated peaks are present in the chromatogram, a model chromatogram of the digests for comparison is absent. The cartridge was connected via a capillary emitter to a mass spectrometer with ESI ionisation source for the analysis of healthy and disease status proteins. A linear gradient from 0 to $60 \%$ acetonitrile was applied with a flow rate of $150 \mathrm{~nL} / \mathrm{min}$. The separation produced three narrow peaks with acceptable resolution, corresponding to the elution of expected proteins.

The pressures achieved in this cartridge system reveal the advances and further potential of electroosmotic pumping systems. The previous system by Ishida was severely limited due to the $<10$ bar operating pressures. Therefore, the system presented here demonstrates substantial progress with respect to the pumping ability, and emphasises the progress towards more economical, compact and efficient pumping systems.

The HPLC cartridge (Fig. 22a) incorporates the majority of HPLC components, excluding the detector, in an assembly weighing $3 \mathrm{~kg}$ and measuring $7000 \mathrm{~cm}^{3}$. Despite lacking an integrated detector, this system satisfies a large proportion of the requirements of a portable HPLC. Gradient generation in a miniaturised device was achieved through exploiting the innately small footprint of EOPs. The cartridge is powered through the HVPS connected to the USB port of a computer. This set up can supply a total of $500 \mathrm{~mA}$, which is more than sufficient for the consumption of the cartridge $(400 \mathrm{~mA}$ at $5 \mathrm{~V}$ ) and uses a novel approach to fulfil the high-power requirements of EOPs.

As stated earlier, the device produced by Ishida et al. possesses the advantage of potentially low fabrication costs of the microfluidic chip-based platform, allowing the overall cost of the system to be kept low. A discussion of the costs of the cartridge was not included by Lynch et al., therefore, while they have stated that a low-cost HPLC cartridge is presented, the cost of the prototype has not been specified.

\section{Chatzimichail et al. (2019)}

As is clear from the instruments reported so far, a major obstacle to the miniaturisation of LC is the pump: there are demanding and simultaneous requirements for it to be small-form factor, achieve high-pressure and support stable flow - and achieve this in very challenging environments outside of a laboratory. Recently, Chatzimichail et al. have explored the controlled expansion of a pre-pressurised gas as the pumping mechanism supporting a field-portable LC. By exploiting the stored energy of the gas, the pumping system does not require electrical power to operate in-situ, thereby making it highly amenable to supporting field-portable LC. It is important to note that the system was the first standalone portable LC that incorporated all chromatographic components and computing units in a single package; the only thing required was the sample itself.

The isocratic UV-absorption HPLC instrument reported was a completely standalone system in a suitcase-style enclosure (Fig. 23a) with dimensions $33 \mathrm{~cm} \times 29 \mathrm{~cm} \times 14 \mathrm{~cm}$ $(\mathrm{L} \times \mathrm{W} \times \mathrm{H})$, and weighed $6.7 \mathrm{~kg}$ with all requisite computer systems, battery units, and solvents fully integrated. The componentry incorporated into the device include a commercially available 6-port injector with a $2 \mu \mathrm{L}$ internal sample injection loop [152]; a guard column positioned between the injection system and flow sensor; a range of easily interchanged RP C18-packed capillary, standard and monolith columns with lengths and I.D.'s ranging from 30 to $150 \mathrm{~mm}$ and $100 \mu \mathrm{m}$ to $1.0 \mathrm{~mm}$, respectively; a commercially available UV-LED light source and miniature spectrometer coupled to a z-type flow cell as the detection system $[153,154]$. The unique pumping mechanism comprised a high pressure (200 bar) $426 \mathrm{~mL}$ air tank pressurised with oxygen-free nitrogen, connected to a $150 \mathrm{~mL}$ mobile phase reservoir using stainless-steel tubing and pipe fittings obtained from Swagelok (Kings Langley, United Kingdom). 
a b

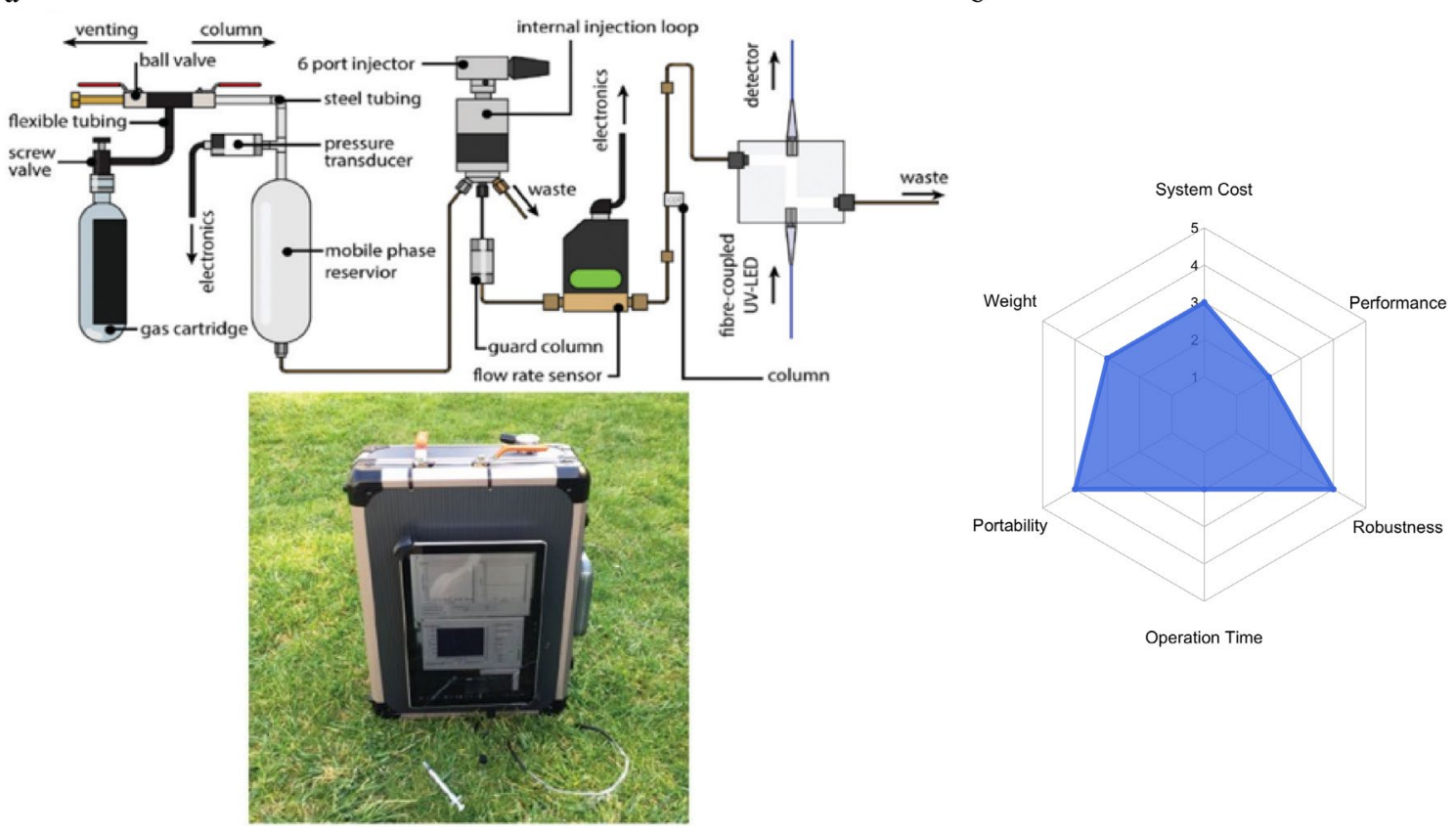

Fig. 23 Portable HPLC suitcase produced by Chatzimichail et al. [66] a Schematic diagram demonstrating system componentry and photograph of the suitcase-based HPLC operating in the field. b Radar chart assessing the system according to the BETTER criteria

Fig. 24 Chromatographic separations performed using the pressurised-gas pump HPLC system produced by Chatzimichail et al. [66] a Pressure and flow rate plots against time demonstrating the almost instant response to pressure changes. b A mixture of the amino acids tryptophan and tyrosine. The mix trace has been shifted vertically to provide clarity a

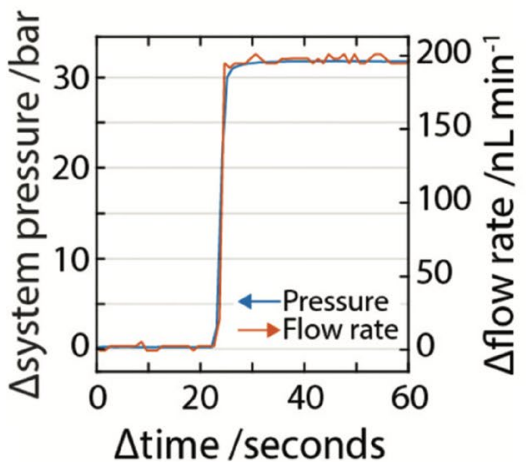

b

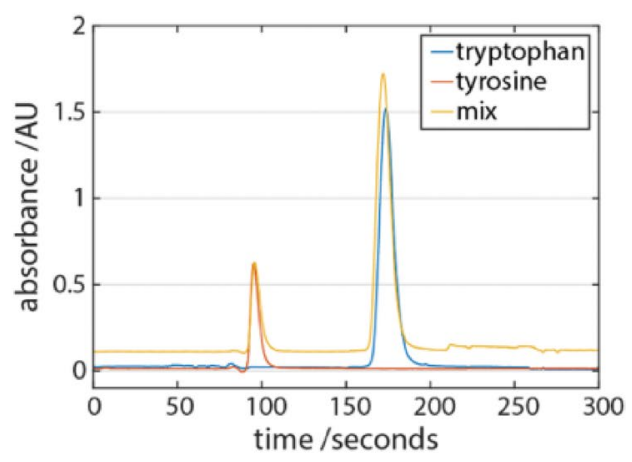

The pump was able to drive stable flow through a range of narrow and microbore columns. An on-board transducer and flow sensor allowed the pressure, and therefore flow rate, to be determined. An increase in pressure in the isocratic system was achieved by opening a valve at the head of the gas cartridge whilst the desired pressure is determined from the pressure transducer's digital output. The pressure changes produced an immediate response in flow rate $(<1 \mathrm{~s})$ (Fig. 24a), demonstrating the feasibility of the pumping mechanism for a range of pressures up to 150 bar and flow

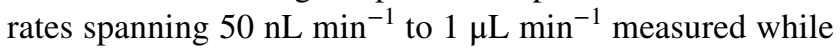
using a range of columns.

The systems analytical performance was demonstrated by separating mixtures of aromatic amino acids and fungicidal pesticides (Fig. 24b). Critical in assessing an instruments performance in the field, measurements were made while the system was using battery-power and outside a laboratory.

Piston pumps commonly used in LC systems are hindered by pulsatile tendencies at low flow rates which appear as baseline variations limiting the system sensitivity. As discussed with previous LC systems, to counteract the pulsations, pulse dampeners are universally employed to reduce the pressure-flow fluctuations with limited success. An advantage of pressure driven flow is the low variation and high stability of flow. The authors confirmed this for their system, reporting a flow precision of $\leq 0.2 \%$ RSD, which is comparable to laboratory based HPLC binary pumps. This was of particular note given the inexpensive nature of the pre-pressurised gas pump. The system that was reported was isocratic. It is worth noting that pressure-driven flow 
can support gradient elution; however, this was not demonstrated. Whilst gradient-based portable LC devices have previously been reported, these, typically, have high power requirements or are expensive.

While the expansion of a pre-pressurised gas requires zero electrical power, the reservoir of gas depletes as it operates, not unlike the charge of a battery. The longevity of the pumping system was estimated such that 128 (1 mm I.D. column) to $13,870(100 \mu \mathrm{m}$ I.D. column $)$ chromatographic runs were able to be supported before the analytical aims of the separation were adversely affected. The ease-of-use of the system may be debated since access to pure nitrogen gas can be limited for researchers conducting field analyses in

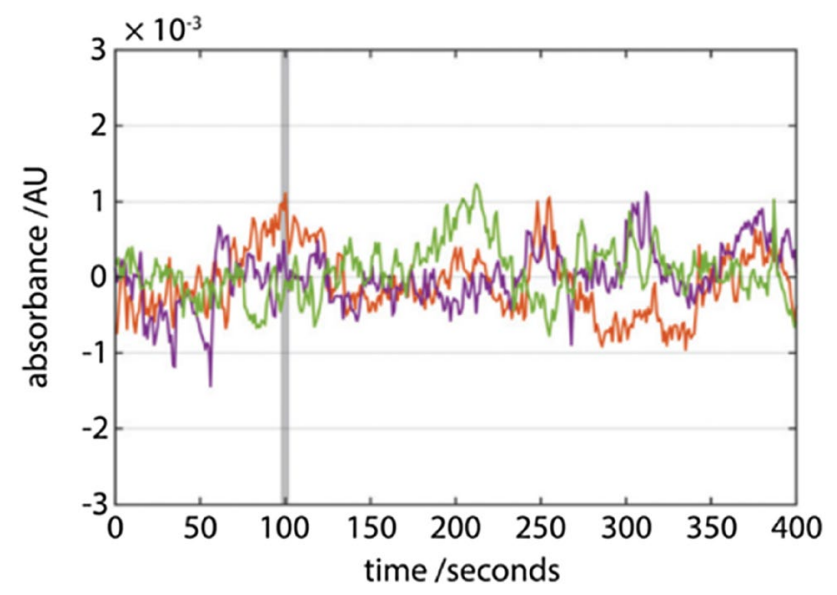

Fig. 25 Mechanical stability of the portable HPLC device by Chatzimichail et al. demonstrated through repeated $1 \mathrm{~m}$ drop tests [66]. Plots show the absorbance $(n=3)$ and the grey vertical bar indicates the approximate time range in which the device is freefalling and impact. Reproduced from Chatzimichail et al. [66] remote areas. It stands to reason that atmospheric gas could be utilised in replacement of a purified gas source; however, this was not considered or tested.

Chatzimichail et al. reasoned that the lack of moving parts of the system would afford it a good degree of mechanical stability. To test this, they performed several drop tests from a vertical height of $1 \mathrm{~m}$ while the device was operating (Fig. 25). These tests confirmed the stability and ruggedness of the device to impact shocks, since there was negligible divergence in the baseline noise prior to and following impact. It is the only report to have characterised a portable LC's mechanical stability despite widespread claims of instruments being ideal for use in the field-testing environment.

\section{Lam et al. (2020)}

Following on from the group's modular LC platform presented by Li et al. in 2015, Lam et al. recently presented an updated prototype [155]. It is noteworthy for its demonstration of hyphenation with a portable mass spectrometer and the in-situ isocratic LC analysis of freeze-dried plant material carried out on a farm site.

The instrument was similar in specification to it's predecessor (Fig. 26): The gradient enabled, capillary LC was housed in a 3D printed box with dimensions of $24.5 \mathrm{~cm} \times 18.5 \mathrm{~cm} \times 16.0 \mathrm{~cm}(\mathrm{~L} \times \mathrm{W} \times \mathrm{H})$ with a total weight of $2.7 \mathrm{~kg}$. The system is predominantly based on $\mu$ Process ${ }^{\mathrm{TM}}$ automated syringe pumps and valves sourced from LabSmith Inc. The pumping system comprised two syringe pumps (SPS01, $20 \mathrm{~mL}$ volume, LabSmith) for the aqueous and organic phases connected to two automated 3-port valves (AV201-C360, LabSmith). The 3-port valves are
Fig. 26 The LabSmith (Livermore, CA, USA) supported portable LC system, showing a (A) arrangement of components [components: (1) LED detector and flow cell setup; (2) Detector controlling unit; (3) Column heater; (4) Temperature control module; (5) LabSmith component controlling interface; (6) Voltaic V88 battery; (7) LabSmith microfluidic system), and (B) portable LC housed in 3D printed assembly. b Radar chart assessing the system according to the BETTER criteria. Reproduced from Lam et al. [155]

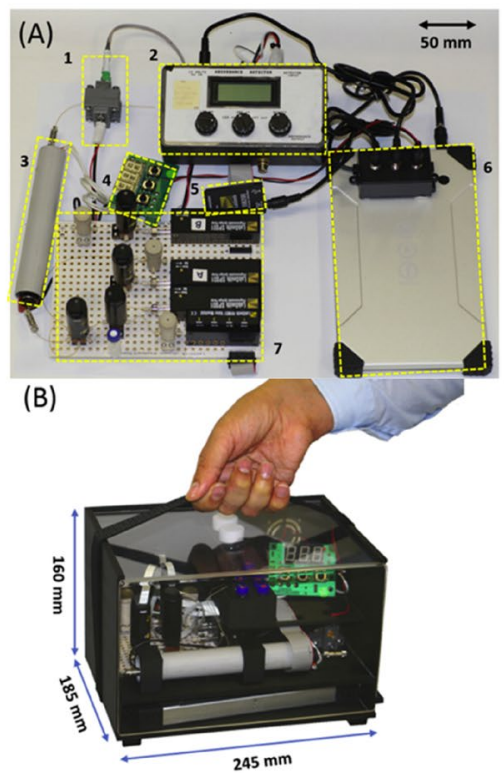

b

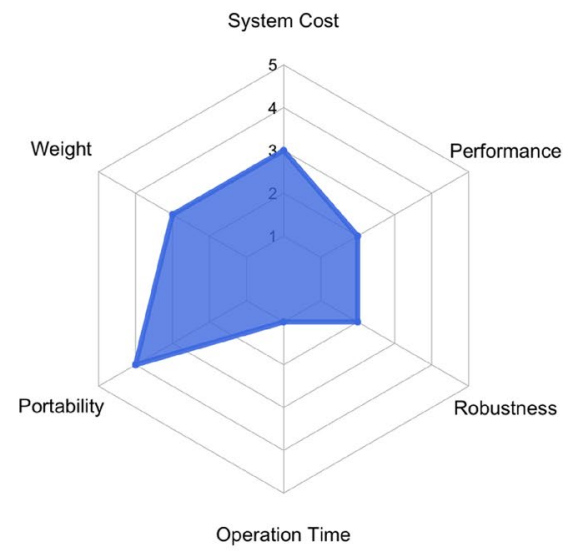


connected to the automated 6-port injection valve (AV303, Labsmith) via a mixing tee, while the sample is introduced via another syringe pump connected to the injection valve using a 3-port valve.

It is not clear why the authors chose to limit the pumping arrangement to 2 syringe pumps to drive solvent, whereas in the previous system 4 were used to enable refilling during gradient operation. As a result, any run is severely restricted to a maximum run time of $8 \mathrm{~min}$. Two self-packed capillary columns $(100 \mathrm{~mm} \times 150 \mathrm{~mm}$ I.D., $5 \mathrm{~mm})(100 \mathrm{~mm} \times 300 \mathrm{~mm}$ I.D., $5 \mathrm{~mm}$ ) were prepared using a MyCapLC ${ }^{\text {TM }}$ kit. The columns were kept at a temperature of $40{ }^{\circ} \mathrm{C}$ using an isothermal aluminium column heater. In combination, these would help lower the back pressure that the syringe pumps would need to overcome. Finally, a $255 \mathrm{~nm}$ UV-LED and photodetector formed part of the detector. The weight $(2.7 \mathrm{~kg})$ of the LC system makes it among the lightest portable LC's to date; however, it must be noted that others include the weights of solvent and computers whereas this does not. The LC system was also demonstrated as a part of a LC-MS system through coupling to a Microsaic 4500 MiD MS system. The performance of the compact system was characterised in the separation of (R)-(-)-1-cyclohexylethyl-amine, sulfamethazine, carbamazepine, ketoprofen and flavone within a separation window of four minutes, showing that despite the restrictions imposed by the syringe pump volumes and limited pressure, fast analyses of pharmaceutical compounds was possible.

\section{Concluding Remarks}

In the past decade, progress in column, pump, injector, and detector technology has spurred on numerous miniaturised systems, with a number of approaches, each of which successfully pushed the boundaries of field-based analytical separations.

Despite this, there are several areas where more focus is required, namely ensuring that instruments offer sufficient performance while doing so in a cost-competitive manner; only then will they find more widespread use at point-ofsampling settings. Further attention is also required with respect to the stability and robustness within field work. Variations in temperature and humidity, as well as shocks and vibrations in the field are unavoidable. The durability of the devices presented in the portable LC field are scarcely discussed. Therefore, specific measures need to be included to ensure the system remains operational; these must also be tested extensively.

The mantra of bringing the laboratory to the sample does well to capture the overall goal of the field. So far, the field has overlooked critical aspects of in-situ analysis. For instance, sample preparation is an integral aspect of LC, particularly for messy field samples, and can be the most time-consuming step of any analysis. How can this be achieved in the field with all the necessary precision to make decisions independent of central laboratories? We must carefully assess whether in-situ field testing can compete with competent infrastructure. The existing approach of sending samples to the laboratory for analysis using laboratory techniques and commercial grade analytical instruments will continue to serve field researchers well. Researchers must carefully evaluate the grounds for challenging such approaches and decide if portable LC can play a meaningful role. Of course, obvious analogies may be drawn from modern day computing and how tasks can be distributed over a range of different devices of varying capability-super computers, desktop workstations, laptops, tablets, mobile phones and even watches. Curiously, the more computationally capable the device, the less often it is used for general tasks; instead tending to be reserved for specialist applications. Will LC and its associated techniques develop a similarly distributed approach?

So far, researchers have focussed on improving specifications to more closely resemble that of their laboratory-based counterparts. Certainly, in doing so this ensures that portable embodiments of LC are not restricted in their applications; however, this can be seen as limiting to their overall development. Indeed, portable systems are for the most part, smaller, simpler variants of conventional instruments. Just as modern mobile phones resemble "traditional" telephones less than early portable models, in the future we are likely to see much more differentiation of instrument form factors as they become more specialised for their intended applications. We predict that innovation will be predominantly driven in the portable LC space as new challenges, unique to point-ofneed in-situ analysis, present themselves.

Moving forward, the portable LC field should focus on determining the defining applications for this technologywhat can portable HPLC accomplish that conventional HPLC simply cannot? In the coming decade, it is likely that the users will answer this in far more interesting ways than the instrument builders have done so far. All in all, this is an important and exciting time for the field.

Acknowledgements This work was supported by the Engineering and Physical Science Research Council (EPSRC) through a fellowship (EP/S001603/2) and Impact Assessment Award (EP/R511559/1) and the Analytical Chemistry Trust Fund (ACTF) through a Community of Analytical Measurement Science (CAMS) Lectureship Award in Analytical Chemistry to ASR, and a KCL NMS faculty studentship awarded to FR. We are grateful to the NIHR Biomedical Facility at Imperial College London for infrastructure support.

Open Access This article is licensed under a Creative Commons Attribution 4.0 International License, which permits use, sharing, adaptation, distribution and reproduction in any medium or format, as long as you give appropriate credit to the original author(s) and the source, 
provide a link to the Creative Commons licence, and indicate if changes were made. The images or other third party material in this article are included in the article's Creative Commons licence, unless indicated otherwise in a credit line to the material. If material is not included in the article's Creative Commons licence and your intended use is not permitted by statutory regulation or exceeds the permitted use, you will need to obtain permission directly from the copyright holder. To view a copy of this licence, visit http://creativecommons.org/licenses/by/4.0/.

\section{References}

1. Salguero FJ, Sánchez-Martín MA, Díaz-San Segundo F et al (2005) Foot-and-mouth disease virus (FMDV) causes an acute disease that can be lethal for adult laboratory mice. Virology 332:384-396. https://doi.org/10.1016/j.virol.2004.11.005

2. Hole K, Nfon C (2019) Foot-and-mouth disease virus detection on a handheld real-time polymerase chain reaction platform. Transbound Emerg Dis 66:1789-1795. https://doi.org/10.1111/ tbed.13227

3. Mobile qPCR Thermocyclers-Biomeme. https://info.biome me.com/mobile-qpcr-thermocyclers. Accessed 11 Mar 2020

4. Siegfried K, Endes C, Bhuiyan AFMK et al (2012) Field testing of arsenic in groundwater samples of Bangladesh using a test kit based on lyophilized bioreporter bacteria. Environ Sci Technol 46:3281-3287. https://doi.org/10.1021/es203511k

5. Arsenator-Digital Arsenic Test Kit-Palintest. https://www. palintest.com/products/arsenator/. Accessed 11 Mar 2020

6. Ellis DI, Muhamadali H, Haughey SA et al (2015) Point-andshoot: rapid quantitative detection methods for on-site food fraud analysis-moving out of the laboratory and into the food supply chain. Anal Methods 7:9401-9414

7. Nunes KM, Andrade MVO, Santos Filho AMP et al (2016) Detection and characterisation of frauds in bovine meat in natura by non-meat ingredient additions using data fusion of chemical parameters and ATR-FTIR spectroscopy. Food Chem 205:14-22. https://doi.org/10.1016/j.foodchem.2016.02.158

8. Downey G (1998) Food and food ingredient authentication by mid-infrared spectroscopy and chemometrics. TrACTrends Anal Chem 17:418-424. https://doi.org/10.1016/S0165 -9936(98)00042-9

9. Rohman A (2019) The employment of Fourier transform infrared spectroscopy coupled with chemometrics techniques for traceability and authentication of meat and meat products. $\mathbf{J}$ Adv Vet Anim Res 6:9-17

10. Rodriguez-Saona LE, Allendorf ME (2011) Use of FTIR for rapid authentication and detection of adulteration of food. Annu Rev Food Sci Technol 2:467-483. https://doi. org/10.1146/annurev-food-022510-133750

11. Handheld FTIR spectrometer-Agilent. https://www.agile nt.com/en/products/ftir/ftir-compact-portable-systems/4300handheld-ftir. Accessed 17 Jan 2020

12. MicroNIR Handheld Spectrometers-VIAVI. https://www. viavisolutions.com/en-uk/osp/products/micronir-spectromet ers/micronir-handheld-products. Accessed 17 Jan 2020

13. Alcalà M, Blanco M, Moyano D et al (2013) Qualitative and Quantitative Pharmaceutical Analysis with a Novel Hand-Held Miniature near Infrared Spectrometer. J Near Infrared Spectrosc 21:445-457. https://doi.org/10.1255/jnirs.1084

14. Handheld Raman Spectrometer-Bruker. https://www.bruke r.com/products/infrared-near-infrared-and-raman-spectrosco py/raman/bravo/overview.html. Accessed 12 Dec 2019

15. Handheld Raman spectrometer-Anton-Paar. https://www. anton-paar.com/uk-en/products/details/handheld-raman-spect rometer-cora-100/. Accessed 12 Dec 2019
16. Portable Raman Spectrometers-BWTEK. https://bwtek.com/ raman-technology/portable/. Accessed 12 Dec 2019

17. Mira DS Handheld Raman Spectrometer-Metrohm. https ://www.metrohm.com/en-gb/products-overview/spectrosco py/mira-handheld-raman-spectrometer/mira-ds-landingpage/\#Form. Accessed 17 Jan 2020

18. Hopkins AJ, Ford AR, Cooper JL, Profeta LTM (2016) Portable deep-ultraviolet (DUV) Raman for standoff detection. Appl Spectrosc 70(5):861-873

19. Ngamsom B, Truyts A, Fourie L et al (2017) A microfluidic device for rapid screening of $E$. coli $\mathrm{O} 157: \mathrm{H} 7$ based on IFAST and atp bioluminescence assay for water analysis. Chem A Eur J 23:12754-12757. https://doi.org/10.1002/chem.201703487

20. Lim JY, Yoon JW, Hovde CJ (2010) A brief overview of Escherichia coli O157:H7 and its plasmid O157. J Microbiol Biotechnol 20:1-10

21. Goesmann F, Brinckerhoff WB, Raulin F et al (2017) The mars organic molecule analyzer (MOMA) instrument: characterization of organic material in martian sediments. Astrobiology 17:655-685. https://doi.org/10.1089/ast.2016.1551

22. Ishida A, Fujii M, Fujimoto T et al (2015) A portable liquid chromatograph with a battery-operated compact electroosmotic pump and a microfluidic chip device with a reversed phase packed column. Anal Sci 31:1163-1169

23. DNA Genetic Testing \& Analysis-23andMe UK. https:// www.23andme.com/en-gb/. Accessed 6 Apr 2020

24. DNA Testing Kits-Living DNA. https://livingdna.com/uk/. Accessed 6 Apr 2020

25. DNA Testing for Ancestry \& Genealogy-FamilyTreeDNA https://www.familytreedna.com/. Accessed 6 Apr 2020

26. DNA Testing-MyHeritage. https://www.myheritage.com/healt h. Accessed 6 Apr 2020

27. DNA Tests for Ethnicity \& Genealogy-AncestryDNA®. https ://www.ancestry.co.uk/dna/. Accessed 6 Apr 2020

28. Stove CP, Ingels A-SME, De Kesel PMM, Lambert WE (2012) Dried blood spots in toxicology: from the cradle to the grave? Crit Rev Toxicol 42:230-243. https://doi.org/10.3109/10408 444.2011.650790

29. Barfield M, Spooner N, Lad R et al (2008) Application of dried blood spots combined with HPLC-MS/MS for the quantification of acetaminophen in toxicokinetic studies. J Chromatogr B Anal Technol Biomed Life Sci 870:32-37. https://doi.org/10.1016/j. jchromb.2008.05.025

30. McDade TW, Williams S, Snodgrass JJ (2007) What a drop can do: dried blood spots as a minimally invasive method for integrating biomarkers into population-based research. Demography 44:899-925

31. Nanthasurasak P, See HH, Zhang M et al (2019) In-transit electroextraction of small-molecule pharmaceuticals from blood. Angew Chemie Int Ed 58:3790-3794. https://doi.org/10.1002/ anie. 201812077

32. Virkler K, Lednev IK (2009) Analysis of body fluids for forensic purposes: from laboratory testing to non-destructive rapid confirmatory identification at a crime scene. Forensic Sci Int 188:1-17

33. Marigo M, Tagliaro F, Poiesi C et al (1986) Determination of morphine in the hair of heroin addicts by high performance liquid chromatography with fluorimetric detection. J Anal Toxicol 10:158-161. https://doi.org/10.1093/jat/10.4.158

34. Achilli G, Cellerino GP, Melzi D’Eril GV, Tagliaro F (1996) Determination of illicit drugs and related substances by highperformance liquid chromatography with an electrochemical coulometric-array detector. J Chromatogr A 729:273-277

35. Floriani G, Gasparetto JC, Pontarolo R, Gonçalves AG (2014) Development and validation of an HPLC-DAD method for simultaneous determination of cocaine, benzoic acid, benzoylecgonine 
and the main adulterants found in products based on cocaine. Forensic Sci Int 235:32-39. https://doi.org/10.1016/j.forsc iint.2013.11.013

36. Molíns Legua C, Campíns Falcó P, Sevillano Cabeza A (1995) Amphetamine and methamphetamine determination in urine by reversed-phase high-performance liquid chromatography with sodium 1,2-naphthoquinone 4-sulfonate as derivatizing agent and solid-phase extraction for sample clean-up. J Chromatogr B Biomed Appl 672:81-88. https://doi.org/10.1016/03784347(95)00201-s

37. Dams R, Benijts T, Lambert WE, De Leenheer AP (2002) Simultaneous determination of in total 17 opium alkaloids and opioids in blood and urine by fast liquid chromatography-diode-array detection-fluorescence detection, after solid-phase extraction. J Chromatogr B Anal Technol Biomed Life Sci 773:53-61. https ://doi.org/10.1016/S1570-0232(01)00594-3

38. Aderjan R, Hofmann S, Schmitt G, Skopp G (1995) Morphine and morphine glucuronides in serum of heroin consumers and in heroin-related deaths determined by hplc with native fluorescence detection. J Anal Toxicol 19:163-168

39. Bhattacharyya SC, Patil RS, Santosh MSSNM et al (2016) A validated reverse phase HPLC technique for the determination of TATB assay. Cent Eur J Energ Mater 13:641-657. https://doi. org/10.22211/cejem/65011

40. Meng HB, Wang TR, Guo BY et al (2008) Simultaneous determination of inorganic anions and cations in explosive residues by ion chromatography. Talanta 76:241-245. https://doi. org/10.1016/j.talanta.2008.01.054

41. Galassi L, Donato R, Tortoli E et al (2003) Nontuberculous mycobacteria in hospital water systems: application of HPLC for identification of environmental mycobacteria. J Water Health 1:133-139. https://doi.org/10.2166/wh.2003.0016

42. Nelson MA, Gates A, Dodlinger M, Hage DS (2004) Development of a portable immunoextraction-reversed-phase liquid chromatography system for field studies of herbicide residues. Anal Chem 76:805-813. https://doi.org/10.1021/ac030298m

43. Johnston EP, Baumann PC (1989) Analysis of fish bile with HPLC - fluorescence to determine environmental exposure to benzo(a)pyrene. Environmental bioassay techniques and their application. Springer, Netherlands, pp 561-566

44. Schroeder DC (1987) The analysis of nitrate in environmental samples by reversed-phase HPLC. J Chromatogr Sci 25:405408. https://doi.org/10.1093/chromsci/25.9.405

45. Monzón AL, Moreno DV, Padrón MET et al (2007) Solidphase microextraction of benzimidazole fungicides in environmental liquid samples and HPLC-fluorescence determination. Anal Bioanal Chem. 387:1957-1963

46. Wang L, Jiang GB, Cai YQ et al (2007) Cloud point extraction coupled with HPLC-UV for the determination of phthalate esters in environmental water samples. J Environ Sci 19:874878. https://doi.org/10.1016/S1001-0742(07)60145-4

47. Sun L, Chen L, Sun X et al (2009) Analysis of sulfonamides in environmental water samples based on magnetic mixed hemimicelles solid-phase extraction coupled with HPLC-UV detection. Chemosphere 77:1306-1312. https://doi.org/10.1016/j. chemosphere.2009.09.049

48. Li J, Cai Y, Shi Y et al (2008) Analysis of phthalates via HPLC-UV in environmental water samples after concentration by solid-phase extraction using ionic liquid mixed hemimicelles. Talanta 74:498-504. https://doi.org/10.1016/j.talan ta.2007.06.008

49. Manual handling at work: a brief guide-HSE UK. https:// www.hse.gov.uk/pubns/indg143.htm. Accessed 6 Apr 2020

50. Irlam RC, Parkin MC, Brabazon DP et al (2019) Improved determination of femtogram-level organic explosives in multiple matrices using dual-sorbent solid phase extraction and liquid chromatography-high resolution accurate mass spectrometry. Talanta 203:65-76. https://doi.org/10.1016/j.talan ta.2019.05.047

51. Salmean C, Dimartino S (2019) 3D-printed stationary phases with ordered morphology: state of the art and future development in liquid chromatography. Chromatographia 82:443-463

52. Adamopoulou T, Deridder S, Desmet G, Schoenmakers PJ (2018) Two-dimensional insertable separation tool (TWIST) for flow confinement in spatial separations. J Chromatogr A 1577:120 123. https://doi.org/10.1016/j.chroma.2018.09.054

53. Welch CJ, Nowak T, Joyce LA, Regalado EL (2015) Cocktail chromatography: enabling the migration of hplc to nonlaboratory environments. ACS Sustain Chem Eng 3:1000-1009. https://doi. org/10.1021/acssuschemeng.5b00133

54. Gaber Y, Törnvall U, Kumar MA et al (2011) HPLC-EAT (Environmental Assessment Tool): a tool for profiling safety, health and environmental impacts of liquid chromatography methods. Green Chem 13:2021-2025. https://doi.org/10.1039/c0gc00667j

55. Baram GI (1996) Portable liquid chromatograph for mobile laboratories. I. Aims. J Chromatogr A 728:387-399. https://doi. org/10.1016/0021-9673(95)01271-0

56. Sharma S, Tolley LT, Tolley HD et al (2015) Hand-portable liquid chromatographic instrumentation. J Chromatogr A 1421:3847. https://doi.org/10.1016/j.chroma.2015.07.119

57. Sharma S, Plistil A, Simpson RS et al (2014) Instrumentation for hand-portable liquid chromatography. J Chromatogr A 1327:80-89. https://doi.org/10.1016/j.chroma.2013.12.059

58. Sharma S, Plistil A, Barnett HE et al (2015) Hand-portable gradient capillary liquid chromatography pumping system. Anal Chem 87:10457-10461. https://doi.org/10.1021/acs.analc hem. 5 b02583

59. Lynch KB, Chen A, Yang Y et al (2017) High-performance liquid chromatographic cartridge with gradient elution capability coupled with UV absorbance detector and mass spectrometer for peptide and protein analysis. J Sep Sci 40:2752-2758. https://doi. org/10.1002/jssc. 201700185

60. Leach AM, Wheeler AR, Zare RN (2003) Flow injection analysis in a microfluidic format. Anal Chem 75:967-972. https://doi. org/10.1021/ac0261121

61. Thurmann S, Lotter C, Heiland JJ et al (2015) Chip-based highperformance liquid chromatography for high-speed enantioseparations. Anal Chem 87:5568-5576. https://doi.org/10.1021/acs. analchem.5b00210

62. Unger MA (2000) Monolithic microfabricated valves and pumps by multilayer soft lithography. Science 288:113-116. https://doi. org/10.1126/science.288.5463.113

63. Ogden S, Bodén R, Hjort K (2010) A latchable valve for highpressure microfluidics. J Microelectromechanical Syst 19:396401. https://doi.org/10.1109/JMEMS.2010.2041749

64. Sharma G, Svensson S, Ogden S et al (2011) High-pressure stainless steel active membrane microvalves-IOPscience. J Micromech Microeng 21:075010

65. Li Y, Dvořák M, Nesterenko PN et al (2015) Miniaturised medium pressure capillary liquid chromatography system with flexible open platform design using off-the-shelf microfluidic components. Anal Chim Acta 896:166-176. https://doi. org/10.1016/j.aca.2015.09.015

66. Chatzimichail S, Casey D, Salehi-Reyhani A (2019) Zero electrical power pump for portable high-performance liquid chromatography. Analyst. https://doi.org/10.1039/C9AN01302D

67. Gu C, Jia Z, Zhu Z et al (2012) Miniaturized electroosmotic pump capable of generating pressures of more than 1200 bar. Anal Chem 84:57. https://doi.org/10.1021/ac3025703

68. Li Z, Mak SY, Sauret A, Shum HC (2014) Syringe-pump-induced fluctuation in all-aqueous microfluidic system implications 
for flow rate accuracy. Lab Chip 14:744-749. https://doi. org/10.1039/c3lc51176f

69. Wang X, Cheng C, Wang S, Liu S (2009) Electroosmotic pumps and their applications in microfluidic systems. Microfluid Nanofluidics 6:145-162

70. Lazar IM, Karger BL (2002) Multiple open-channel electroosmotic pumping system for microfluidic sample handling. Anal Chem 74:6259-6268. https://doi.org/10.1021/ac0203950

71. Dasgupta PK, Liu S (1994) Electroosmosis: a reliable fluid propulsion system for flow injection analysis. Anal Chem 66:17921798. https://doi.org/10.1021/ac00083a004

72. Piette V, Lammerhofer M, Lindner W, Crommen J (1999) Enantiomeric separation of $\mathrm{N}$-protected amino acids by nonaqueous capillary electrophoresis using quinine or Tert-butyl carbamoylated quinine as chiral additive. Chirality 11:622-630. https://doi.org/10.1002/(SICI)1520-636X(1999)11:8<622:AIDCHIR3 > 3.0.CO;2-P

73. Piette V, Lindner W, Crommen J (2002) Enantiomeric separation of N-protected amino acids by non-aqueous capillary electrophoresis with dimeric forms of quinine and quinidine derivatives serving as chiral selectors. J Chromatogr A 948:295-302

74. Chankvetadze B, Blaschke G (2000) Enantioseparations using capillary electromigration techniques in nonaqueous buffers. Electrophoresis 21:4159-4178. https://doi.org/10.1002/15222683(200012)21:18<4159:AID-ELPS4159>3.0.CO;2-G

75. Servais A, Fillet M, Chiap P et al (2002) Optimization of the separation of $\beta$-blockers by ion-pair capillary electrophoresis in nonaqueous media using univariate and multivariate approaches. $\mathbf{J}$ Sep Sci 25:1087-1095. https://doi.org/10.1002/1615-9314(20021 101) 25:15/17<1087:AID-JSSC1087>3.0.CO;2-A

76. Servais A-C, Rousseau A, Fillet M et al (2010) Separation of propranolol enantiomers by $\mathrm{CE}$ using sulfated $\beta-\mathrm{CD}$ derivatives in aqueous and non-aqueous electrolytes: comparative $\mathrm{CE}$ and NMR study. Electrophoresis. https://doi.org/10.1002/elps.20090 0738

77. Chankvetadze L, Servais AC, Fillet M et al (2012) Comparative enantioseparation of talinolol in aqueous and non-aqueous capillary electrophoresis and study of related selector-selectand interactions by nuclear magnetic resonance spectroscopy. J Chromatogr A 1267:206-216. https://doi.org/10.1016/j.chrom a.2012.08.063

78. Sandra K, Vandenbussche J, T'Kindt R et al (2017) Evaluation of micro-pillar array columns ( $\mu$ PAC) combined with high resolution mass spectrometry for lipidomics. Spec Issues 30:6-13

79. Micro-Pillar Array Columns-PharmaFluidics. https://www. pharmafluidics.com/our-products/\#1479749235626-2c3347278a4a. Accessed 17 Jan 2020

80. Fee C, Nawada S, Dimartino S (2014) 3D printed porous media columns with fine control of column packing morphology. J Chromatogr A 1333:18-24. https://doi.org/10.1016/j.chrom a.2014.01.043

81. Dolamore F, Dimartino S, Fee CJ (2019) Numerical elucidation of flow and dispersion in ordered packed beds: nonspherical polygons and the effect of particle overlap on chromatographic performance. Anal Chem 91:15009-15016. https://doi.org/10.1021/ acs.analchem. 9 b03598

82. Jehlička J, Culka A, Bersani D, Vandenabeele P (2017) Comparison of seven portable Raman spectrometers: beryl as a case study. J Raman Spectrosc 48:1289-1299. https://doi.org/10.1002/ jrs.5214

83. Fujihara J, Fujita Y, Yamamoto T et al (2017) Blood identification and discrimination between human and nonhuman blood using portable Raman spectroscopy. Int J Legal Med 131:319322. https://doi.org/10.1007/s00414-016-1396-2
84. Ali EMA, Edwards HGM (2017) The detection of flunitrazepam in beverages using portable Raman spectroscopy. Drug Test Anal 9:256-259. https://doi.org/10.1002/dta.1969

85. Zou Y, Huang M, Wang K et al (2016) Urine surface-enhanced Raman spectroscopy for non-invasive diabetic detection based on a portable Raman spectrometer. Laser Phys Lett. https://doi. org/10.1088/1612-2011/13/6/065604

86. Tondepu C, Toth R, Navin CV et al (2017) Screening of unapproved drugs using portable Raman spectroscopy. Anal Chim Acta 973:75-81. https://doi.org/10.1016/j.aca.2017.04.016

87. Navin CV, Tondepu C, Toth R et al (2017) Quantitative determinations using portable Raman spectroscopy. J Pharm Biomed Anal 136:156-161. https://doi.org/10.1016/j.jpba.2016.12.020

88. Wang G, Xia G, Li Z et al (2018) Design and key technology research of portable UV-VIS spectrometer. Opto-Electronic Eng. https://doi.org/10.12086/oee.2018.180195

89. Scheeline A, Bùi TA (2016) Stacked, mutually rotated diffraction gratings as enablers of portable visible spectrometry. Appl Spectrose 70:766-777

90. UV-VIS Spectrophotometer-Vernier. https://www.vernier.com/ products/sensors/spectrometers/ultraviolet-range/vsp-uv/. Accessed 6 Jan 2020

91. Portable UV-VIS Spectrometer with LCD screen-Allied Scientific Pro. https://alliedscientificpro.com/shop/product/portablespectrometer-uv-vis-with-lcd-screen-19311. Accessed 6 Jan 2020

92. Shibayama K, Yokino T, Hikita K, et al (2009) Miniaturized UV/VIS spectrometer made by MOEMS technology. In: Dickensheets DL, Schenk H, Piyawattanametha W (eds). Proc. 7208, MOEMS and Miniaturized Systems VIII, 720803, p 720803. https://doi.org/10.1117/12.808898

93. Correia RM, Domingos E, Cáo VM et al (2018) Portable near infrared spectroscopy applied to fuel quality control. Talanta 176:26-33. https://doi.org/10.1016/j.talanta.2017.07.094

94. Correia RM, Domingos E, Tosato F et al (2018) Portable near infrared spectroscopy applied to abuse drugs and medicine analyses. Anal Methods 10:593-603. https://doi.org/10.1039/ c7ay02998e

95. Leung Tang P, McCumskay R, Rogerson M et al (2019) Handheld FT-IR spectroscopy for the triage of micro- and mesosized plastics in the marine environment incorporating an accelerated weathering study and an aging estimation. Spectroscopy 34:54-60

96. Karunathilaka SR, Mossoba MM, Chung JK et al (2017) Rapid prediction of fatty acid content in marine oil omega-3 dietary supplements using a portable fourier transform infrared (FTIR) device and partial least-squares regression (PLSR) analysis. J Agric Food Chem 65:224-233. https://doi.org/10.1021/acs. jafc.6b04463

97. Wasson A, Bischof L, Zwart A, Watt M (2016) A portable fluorescence spectroscopy imaging system for automated root phenotyping in soil cores in the field. J Exp Bot 67:1033-1043. https://doi.org/10.1093/jxb/erv570

98. Dos Santos CH, Romano RA, Nicolodelli G et al (2015) Performance evaluation of a portable laser-induced fluorescence spectroscopy system for the assessment of the humification degree of the soil organic matter. J Braz Chem Soc 26:775783. https://doi.org/10.5935/0103-5053.20150039

99. Mu T, Chen S, Zhang Y et al (2016) Portable detection and quantification of olive oil adulteration by 473-nm laser-induced fluorescence. Food Anal Methods 9:275-279. https://doi. org/10.1007/s12161-015-0199-2

100. Zhou S, Yuan Z, Cheng Q et al (2019) Quantitative analysis of iron and silicon concentrations in iron ore concentrate using portable x-ray fluorescence (XRF). Appl Spectrosc. https://doi. org/10.1177/0003702819871627 
101. Sun F, Bakr N, Dang T et al (2020) Enhanced soil profile visualization using portable X-ray fluorescence (PXRF) spectrometry. Geoderma. https://doi.org/10.1016/j.geoderma.2019.11399 7

102. Ravansari R, Wilson SC, Tighe M (2020) Portable X-ray fluorescence for environmental assessment of soils: not just a point and shoot method. Environ Int 134:105250

103. Guijt RM, Manz A (2018) Miniaturised total chemical-analysis systems (MTAS) that periodically convert chemical into electronic information. Sens Actuators B Chem 273:1334-1345

104. Chervet JP, Van Soest REJ, Ursem M (1991) Z-shaped flow cell for UV detection in capillary electrophoresis. J Chromatogr A 543:439-449. https://doi.org/10.1016/S0021-9673(01)95795-4

105. Dolan JW (2016) How does it work? Part IV: ultraviolet detectors. LCGC North Am 34:534-539

106. Dolan JW (2014) UV detector problems. LCGC North Am $32: 404-409$

107. Sharma S, Dennis Tolley H, Farnsworth PB, Lee ML (2014) LED-based UV absorption detector with low detection limits for capillary liquid chromatography. Anal Chem 87:1381-1386. https://doi.org/10.1021/ac504275m

108. Capitán-Vallvey LF, Palma AJ (2011) Recent developments in handheld and portable optosensing - a review. Anal Chim Acta 696:27-46

109. Leigh RJ, Whyte C, Cutter MA et al (2017) ComPAQS: a compact concentric UV/visible spectrometer, providing a new tool for air quality monitoring from space. In: Costeraste J, Armandillo E, Karafolas N (eds) International conference on space opticsICSO 2008. SPIE, Bellingham, p 52

110. Pena-Pereira F, Costas-Mora I, Romero V et al (2011) Advances in miniaturized UV-Vis spectrometric systems. TrAC-Trends Anal Chem 30:1637-1648

111. Grasse EK, Torcasio MH, Smith AW (2016) Teaching UV-Vis spectroscopy with a 3D-printable smartphone spectrophotometer. J Chem Educ 93:146-151. https://doi.org/10.1021/acs. jchemed.5b00654

112. Mach PM, McBride EM, Sasiene ZJ et al (2015) Vehiclemounted portable mass spectrometry system for the covert detection via spatial analysis of clandestine methamphetamine laboratories. Anal Chem 87:11501-11508. https://doi. org/10.1021/acs.analchem.5b03269

113. Pulliam CJ, Bain RM, Wiley JS et al (2015) Mass spectrometry in the home and garden. J Am Soc Mass Spectrom 26:224-230. https://doi.org/10.1007/s13361-014-1056-z

114. Chen $\mathrm{CH}$, Chen TC, Zhou X et al (2015) Design of portable mass spectrometers with handheld probes: aspects of the sampling and miniature pumping systems. J Am Soc Mass Spectrom 26:240-247. https://doi.org/10.1007/s13361-014-1026-5

115. Gómez-Ríos GA, Vasiljevic T, Gionfriddo E et al (2017) Towards on-site analysis of complex matrices by solid-phase microextraction-transmission mode coupled to a portable mass spectrometer: via direct analysis in real time. Analyst 142:2928-2935. https://doi.org/10.1039/c7an00718c

116. Snyder DT, Pulliam CJ, Ouyang Z, Cooks RG (2016) Miniature and fieldable mass spectrometers: recent advances. Anal Chem 88:2-29

117. Zhai Y, Feng Y, Wei Y et al (2015) Development of a miniature mass spectrometer with continuous atmospheric pressure interface. Analyst 140:3406-3414. https://doi.org/10.1039/c5an0 $0462 \mathrm{~d}$

118. Li L, Chen TC, Ren Y et al (2014) Mini 12, miniature mass spectrometer for clinical and other applications-introduction and characterization. Anal Chem 86:2909-2916. https://doi. org/10.1021/ac403766c

119. Gerbig S, Neese S, Penner A et al (2017) Real-time food authentication using a miniature mass spectrometer. Anal
Chem 89:10717-10725. https://doi.org/10.1021/acs.analc hem.7b01689

120. Pulliam CJ, Bain RM, Osswald HL et al (2017) Simultaneous online monitoring of multiple reactions using a miniature mass spectrometer. Anal Chem 89:6969-6975. https://doi.org/10.1021/ acs.analchem.7b00119

121. Zou R, Cao W, Chong L et al (2019) Point-of-care tissue analysis using miniature mass spectrometer. Anal Chem 91:1157-1163. https://doi.org/10.1021/acs.analchem.8b04935

122. Edwards E, Thomas-Oates J (2005) Hyphenating liquid phase separation techniques with mass spectrometry: on-line or offline. Analyst 130:13-17

123. Zhao X, Xie X, Sharma S et al (2017) Compact ultrahighpressure nanoflow capillary liquid chromatograph. Anal Chem 89:807-812. https://doi.org/10.1021/acs.analchem.6b03575

124. Giannoukos S, Brkić B, Taylor S, France N (2014) Monitoring of human chemical signatures using membrane inlet mass spectrometry. Anal Chem 86:1106-1114. https://doi.org/10.1021/ ac403621c

125. Giannoukos S, Brkić B, Taylor S, France N (2015) Membrane inlet mass spectrometry for homeland security and forensic applications. J Am Soc Mass Spectrom 26:231-239. https://doi. org/10.1021/JASMS.8B04965

126. Guo Q, Gao L, Zhai Y, Xu W (2018) Recent developments of miniature ion trap mass spectrometers. Chin Chem Lett 29:15781584. https://doi.org/10.1016/j.cclet.2017.12.009

127. Awad H, El-Aneed A (2013) Enantioselectivity of mass spectrometry: challenges and promises. Mass Spectrom Rev $32: 466-483$

128. Tomer KB (2001) Separations combined with mass spectrometry. Chem Rev 101:297-328. https://doi.org/10.1021/cr990091m

129. Lubda D, Mueller E (2007) Method for producing monolithic chromatography columns. US Patent 7,235,199 B2

130. Malik A, Wang D (2007) Capillary column and method of making. US Patent 2007/017296 A1

131. Cielecka-Piontek J, Zalewski P, Jelińska A, Garbacki P (2013) UHPLC: the greening face of liquid chromatography. Chromatographia 76:1429-1437

132. Modlin DN, Chazan D (2005) Microfluidic system with integrated permeable membrane. US Patent 205/026582 A1

133. Killinger D, Sharikova A, Sivaprakasam V (2012) Deep-UV led and laser induced fluorescence detection and monitoring of trace organics in potable liquids. US Patent 2012/0001094 A1

134. Otagawa T, Stetter JR, Zaromb S (1986) Portable liquid chromatograph for analysis of primary aromatic amines in coal-derived materials. J Chromatogr A 360:252-259. https://doi.org/10.1016/ S0021-9673(00)91671-6

135. Hamilton RJ, Sewell PA (1982) Introduction to high performance liquid chromatography. Introduction to high performance liquid chromatography, 2nd edn. Springer, Netherlands, Dordrecht, pp 60-61

136. Baram GI, Grachev MA, Komarova NI et al (1983) Micro-column liquid chromatography with multi-wave-length photometric detection. I. The OB-4 micro-column liquid chromatograph. J Chromatogr A 264:69-90. https://doi.org/10.1016/S0021 -9673(01)95007-1

137. Tulchinsky VM, Glazunov LL, Karev VV, Morozova ER (1997) Portable HPLC - new generation of high performance liquid chromatographs. In: Gottlieb J, Hötzl H, Huck KNR (eds) Field screening Europe. Springer, Netherlands, Dordrecht, pp 367-370

138. Tulchinsky VM, St. Angelo DE (1998) A practical portable HPLC system-MINICHROM, a new generation for field HPLC. F Anal Chem Technol 2:281-285. https://doi.org/10.1002/ (SICI)1520-6521(1998)2:5<281:AID-FACT5>3.0.CO;2-V 
139. Lynch KB, Chen A, Liu S (2018) Miniaturized high-performance liquid chromatography instrumentation. Talanta 177:94-103. https://doi.org/10.1016/j.talanta.2017.09.016

140. Woolley AT, Lao K, Glazer AN, Mathies RA (1998) Capillary electrophoresis chips with integrated electrochemical detection. Anal Chem 70:684-688. https://doi.org/10.1021/ac971135z

141. Wang J, Tian B, Sahlin E (1999) Micromachined electrophoresis chips with thick-film electrochemical detectors. Anal Chem 71:5436-5440. https://doi.org/10.1021/ac990807d

142. Martin RS, Gawron AJ, Lunte SM, Henry CS (2000) Dualelectrode electrochemical detection for poly(dimethylsiloxane)fabricated capillary electrophoresis microchips. Anal Chem 72:3196-3202. https://doi.org/10.1021/ac000160t

143. Zotou A (2012) An overview of recent advances in HPLC instrumentation. Cent Eur J Chem 10:554-569

144. Mao X, Huang TJ (2012) Microfluidic diagnostics for the developing world. Lab Chip 12:1412-1416

145. LabSmith. https://products.labsmith.com/. Accessed 22 Nov 2019

146. Miniature Precision Fluid Control-The Lee Company. https:// www.theleeco.com/. Accessed 22 Nov 2019

147. SCIEX. https://sciex.com/. Accessed 22 Nov 2019

148. He C, Zhu Z, Gu C et al (2012) Stacking open-capillary electroosmotic pumps in series to boost the pumping pressure to drive high-performance liquid chromatographic separations. J Chromatogr A 1227:253-258. https://doi.org/10.1016/j.chrom a.2011.12.105

149. Zhou L, Lu JJ, Gu C, Liu S (2014) Binary electroosmotic-pump nanoflow gradient generator for miniaturized high-performance liquid chromatography. Anal Chem 86:12214-12219. https://doi. org/10.1021/ac503223r

150. Chen A, Lu JJ, Gu C et al (2015) Combining selection valve and mixing chamber for nanoflow gradient generation: toward developing a liquid chromatography cartridge coupled with mass spectrometer for protein and peptide analysis. Anal Chim Acta 887:230-236. https://doi.org/10.1016/j.aca.2015.06.035

151. Chen A, Lynch KB, Wang X et al (2014) Incorporating highpressure electroosmotic pump and a nano-flow gradient generator into a miniaturized liquid chromatographic system for peptide analysis. Anal Chim Acta 844:90-98. https://doi.org/10.1016/j. aca.2014.06.042

152. Rheodyne 7725-IDEX Corporation, USA. https://www.idex-hs. $\mathrm{com} /$ store/fluidics/valves/analytical-front-loading-injection-valve .html. Accessed 1 Dec 2019

153. UV Light Sources-Ocean Insight. https://www.oceaninsig ht.com/products/light-sources/. Accessed 1 Dec 2019

154. UV-NIR Spectrometer-Ocean Insight. https://www.oceaninsig ht.com/products/spectrometers/usb-series/usb-uv-nir/. Accessed 1 Dec 2019

155. Lam SC, Coates LJ, Hemida M et al (2020) Miniature and fully portable gradient capillary liquid chromatograph. Anal Chim Acta 1101:199-210. https://doi.org/10.1016/j.aca.2019.12.014

Publisher's Note Springer Nature remains neutral with regard to jurisdictional claims in published maps and institutional affiliations. 